

\section{DISCLAIMER}

This report was prepared as an account of work sponsored by an agency of the United States Government. Neither the United States Government nor any agency Thereof, nor any of their employees, makes any warranty, express or implied, or assumes any legal liability or responsibility for the accuracy, completeness, or usefulness of any information, apparatus, product, or process disclosed, or represents that its use would not infringe privately owned rights. Reference herein to any specific commercial product, process, or service by trade name, trademark, manufacturer, or otherwise does not necessarily constitute or imply its endorsement, recommendation, or favoring by the United States Government or any agency thereof. The views and opinions of authors expressed herein do not necessarily state or reflect those of the United States Government or any agency thereof. 


\section{DISCLAIMER}

Portions of this document may be illegible in electronic image products. Images are produced from the best available original document. 
This document contains Secret-Restricted Data relating to civilian applications of atomic energy.

\section{LEGAL NOTICE}

This report was prepared as an account of Government sponsored work. Neither the United States, nor the Commission, nor any person acting on behalf of the Commission:

A. Makes any warranty or representation, express or implied, with respect to the accuracy, completeness, or usefulness of the information contained in this report, or that the use of any information, apparatus, method, or process disclosed in this report may not infringe privately owned rights; or

B. Assumes any liabilities with respect to the use of, or for damages resulting from the use of any information, apparatus, method, or process disclosed in this report.

As used in the above, "person acting on behalf of the Commission" includes any employee or contractor of the Commission to the extent that such employee or contractor prepares, handles or distributes, or provides access to, any information pursuant to his employment or contract with the Commission. 
UCR L-4996

Nuclear Rocket and

Ram-jet Engines

-

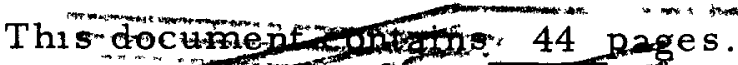

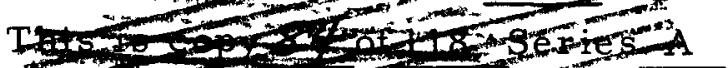

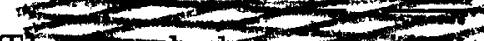

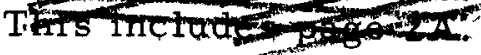

\author{
UNIVERSIT Y OF CALIFORNIA \\ Radiation Laboratory, Livermore Site \\ Livermore, California
}

Contract No. W-7405-eng-48

\title{
A STUDY OF THE NUCLEAR GASEOUS REACTOR ROCKET
}

Robert H. Fox

October 31,1957

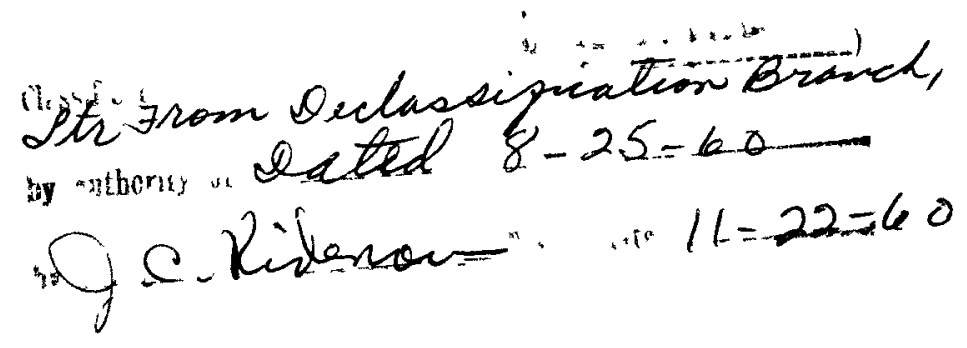

wimedocument contains restricted as defined in tyromic Energy Act of

1954. Its traptsolat or the of its congus in any manner to an unavurorizedperson is prohibited.

Printed for the U. S. Atomic Energy Commission 


\section{DISTRIBUTION}

Series A

Copy No.

UCR L Livermore: Information Division

Herbert F. York

T. C. Merkle

Robert H. Fox

Arthur Maimoni

Robert Meuser

UCRL Berkeley: R. K. Wakerling

Aircraft Reactor Br., Division of Reactor Development

Aerojet-General Corporation, Azusa

Rocketdyne, North American Aviation, Canoga Park

Oak Ridge National Laboratory

The $R$ and Corporation

Air Force Ballistic Missile Division

$A F P R$, Douglas, Long Beach

AFPR, North American, Canoga Park

AFPR, North American, Downey

Air Force Special Weapons Center

Air Research and Development command (RDGN)

Air Research and Development Command (RDTAPS)

Air Research and Development Command (RDZPSP)

Air Technical Intelligence Center

ANP Project Office, Convair, Fort Worth

Albuquerque Operations Office

Armed Forces Special Weapons Project, Sandia

Armed Forces Special Weapons Project, Washington

Army Ballistic Missile Agency

Assistant Secretary of Defense, R \& D (WSEG)

Atomic Energy Commission, Washington

Atomics International

Bureau of Aeronautics

BAR, Aerojet-General, Azusa 


\section{DISTRIBUTION (Contd.)}

Series A

Copy No.

BAR, Glenn L. Martin, Baltimore 56

$\begin{array}{ll}\text { Bureau of Ordnance } & 57\end{array}$

Bureau of Ordnance (SP-209) 58

Bureau of Ships

General Electric Company (ANPD) $\quad 60$

Jet Propulsion Laboratory 61

Los Alamos Scientific Laboratory $\quad 62-63$

Marquardt Aircraft Company $\quad 64$

National Advisory Committee for Aeronautics, Cleveland 65

National Advisory Committee for Aeronautics, Washington 66

$\begin{array}{ll}\text { Office of Naval Research } & 67\end{array}$

Office of the Assistant for Operations Analysis DCS/O 68

Office of the Chief of Naval Operations (Op-361) 69

$\begin{array}{ll}\text { Office of the Chief of Ordnance } & 70\end{array}$

Patent Branch, Washington $\quad 71$

Redstone Arsenal $\quad 72$

$\begin{array}{ll}\text { San Francisco Operations Office } & 73\end{array}$

Union Carbide Nuclear Company (ORNL)

USAF Project RAND $\quad 75-76$

U. S. Naval Ordnance Test Station $\quad 77$

Wright Air Development Center (WCOSI-3) 78-83

Technical Information Service Extension, Oak Ridge 84-118
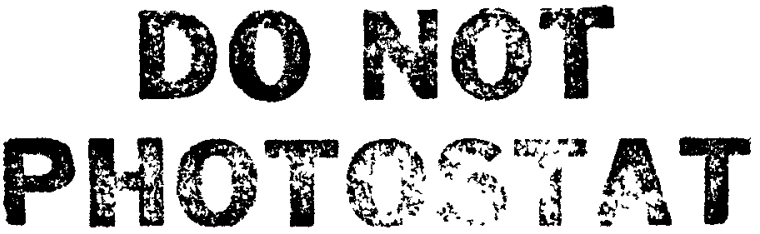
A STUDY OF THE INUCLEAR GASEOUS REACTOR ROCKET

\section{Contents}

\begin{tabular}{|c|c|c|}
\hline & & Page \\
\hline & Abstract & 4 \\
\hline I & INTRODUCTION & 5 \\
\hline II & THE THROUGHPUT CONCEPT & 6 \\
\hline III & SIGNIFICANCE OF THE THROUGHPUT REQUIREMENT & 8 \\
\hline IV & VARIOUS MEANS FOR DECREASING $B$ & 10 \\
\hline $\mathrm{V}$ & INCLUSION OF PAYLOAD AND STRUCTURE & 24 \\
\hline VI & MASS EFFICIENCY & 26 \\
\hline VII & BURNUP EFFICIENCY & 29 \\
\hline VIII & DOLIAR ECONOMY & 30 \\
\hline IX & CENTRIFUGAL SEPARATION & 31 \\
\hline $\mathrm{x}$ & CONCLUSION & 36 \\
\hline
\end{tabular}


A STUDY OF THE INUCLEAR GASEOUS REACTOR ROCKET

Robert I. Fox

University of California Radiation Laboratory

Livermore, California

October 31,1957

ABSTRACT

A detailed study is presented of some fundamental problems connected with the realization of a nuclear gaseous reactor rocket engine. The virtue of such a propulsion scheme is that the enerey of the fission fragments is transferred directly to the propellant. Thus, the heat transfer problem of the "conventional" heat exchanger type of nuclear rocket is avoided and very high values of the specific impulse (>1000 sec) might be achieved. The discussion centers mainly around containment of the propellant gases during heat addition, maintaining the nuclecr chain reaction, and an assessment of the principal factors contributing to cost. It is shown that reasonable values of cost per unit payload are to be achieved only by considering payloads about two orders of magnitude larger than any of current interest. Possible means for improving the situation, such as dissociation-recombination effects, pulsed operation, centrifugal separation of nuclear fuel and propellant, etc., are investigated without reaching favorable results. 
A STUDY OF THE NUCLLAZ GSUEOUS REACTOR ROCKET

Rovert i. FOX

University of California Radiation Laboratory

Livermore,' California

October 31, 1957

\section{INTRODUCTION}

In recent years there has been considerable interest in gas reactors as a possible means of achieving more efficient rocket propulsion than is currently thought to be possible with the "conventional" heat exchanger type of nuclear rocket. We conceive a gas reactor rocket motor to consist of a cavity surrounded by some structure which must serve the multiple functions of moderating and reflecting neutrons and containing the gas which flows through the cavity. This gas is principally propellant with sufficient fissionable material mixed in to maintain a critical system. Presumably the propellant is hydrogen. The principal virtue is supposed to be that the fission fragment energy is delivered directly to the gas without the necessity of any intermediate heat transfer process. Since the heat is not flowing from the cavity walls to the gas it seems likely that one could achieve a much higher gas temperature and hence exhaust velocity vefore reaching limitations arising from the properties of the cavity wall material. Thus, even for large final velocities $\left(v_{f}>10^{6} \mathrm{~cm} / \mathrm{sec}\right)$, very reasonable mass ratios might ensue.

The principal difficulty with this idea is that the total consumption of fissionable material is large compared to the critical mass. It is rather easy to obtain estimates like $10^{4} \mathrm{~kg}$ of fissionable material required for $\mathrm{v}_{\mathrm{f}} \simeq 10^{6} \mathrm{~cm} / \mathrm{sec}$ under any conditions but optimal ones. On the other hand, attempts to reduce the fissionable material requirement usually lead to rather impractical size or weight requirements.

RESTRICTED DATA

This document contains restricted data as defined in the Atomic Energy Act of 1954. Its transmittal or the disclosure of its contents in any manner to an unauthorized person is prohibited. 
The problem is sufficiently complicated so that "back of an envelope" type of calculations have not succeeded in showing clearly either the feasibility or non-feasibility of this type of scheme. As a result, there have been intermittent attempts at this laboratory to analyze this problem from various points of view with the hope of discovering relationships of a fundamental and clear nature which would provide the needed answers or indicate a more fruitful avenue of approach. The purpose of this report is to show what has been accomplished along these lines in recent months. Some rather interesting ideas have developed and will be discussed here. As will be seen, the detailed and difficult problems such as calculation of the critical mass, heat transfer limitations at elevated temperatures, and reactor control (delayed neutrons are lost to the exhaust stream) are avoided. It did not seem wise to spend appreciable effort on these topics unless their solution was found to be the only remaining obstacle to demonstrating feasibility. Further, if the latter situation could be shown to exist, even under optimistic assumptions, one could probably justify a low-level experimental program for their investigation.

\section{THE THROUGHPUT CONCEPT}

Let us first consider the simplest possible system consisting only of a spherical containing shell of mass $M_{S}$ and radius $R$, and a weight of propellant $\mathrm{M}_{f}$, Fig. 1. For the moment we shall ignore nozzle, pump, tank, payload and other miscellaneous weights. Further, we shall assume steadystate operation. Gravitational effects are to be ignored. We now ask how many times must the shell be filled with propellant gas, heated to some temperature $T$, and expelled before the pressure shell reaches a final velocity $V_{f}$. That is, we want to know the number $N$ of throughputs required.

If the propellant is a perfect gas of molecular weight $W$, then from the equation of state and for a pressure $p$ we find that the weight of gas contained at any moment is (assuming homogeneity):

$$
M_{g}=4 / 3 \pi R^{3} \frac{W p}{R_{0} T} \quad R_{0}=\text { universal gas constant }
$$

The available energy (enthalpy) contained in this gas is just

$$
E_{g}=\frac{R_{0} T}{w} \frac{\gamma}{\gamma-1} M_{g}=4 / 3 \pi \frac{\gamma}{\gamma-1} R^{3} p, \quad \gamma=\frac{C p}{C v}
$$


If the density and strength of the pressure shell material are $\rho_{s}$ and $\sigma_{s}$ respectively, then the minimum mass this shell may have without failing is

$$
\mathrm{M}_{\mathrm{s}} \geq 2 \pi \frac{\rho_{\mathrm{s}}}{\sigma_{\mathrm{s}}} \mathrm{R}_{\mathrm{p}}=3 / 2 \frac{\gamma-1}{\gamma} \frac{\rho_{\mathrm{s}}}{\sigma_{\mathrm{s}}} \mathrm{E}_{\mathrm{g}} .
$$

The equality sign holds if the thin shell approximation is valid. Now, from conservation of energy we have

$$
M_{s} \frac{v_{f}^{2}}{2}=\eta N E_{g} . \quad \eta<1
$$

$\eta$ is the fraction of the total energy delivered to the gas which ends up as pressure shell kinetic energy (as seen by an observer in the original inertial frame of reference). We shall call $\eta$ the efficiency of the rocket. Substituting (3) into (4) we have

$$
N \geqslant 3 / 4 \frac{\gamma-1}{\gamma} \frac{\rho_{s}}{\eta \sigma s} v_{f}^{2} \equiv \frac{B}{\eta} v_{f}^{2}
$$

Thus, simply by requiring energy conservation and containment of the gas during heat addition we have found a general characteristic of rocket motors. Note that this result is just as applicable to a heat exchanger rocket as it is to the specific type being considered here. In fact, this result applies to all systems in which the gas is contained in a pressure shell during the heat addition process, whether pulsed or steady state. The constant B will, of course, vary somewhat from one specific case to another, and this variation will be investigated in the remainder of the report.

Before proceeding further, let us look briefly at the values for $\eta$ which we may expect to obtain. We have

$$
\eta=\eta_{1}
$$

where $\eta_{1}$ is the ratio of the mechanical energy given to the exhaust gases $\left(M_{f} v / 2\right)$ and the final kinetic energy of the rocket at burnout $\left(M_{s} v_{f}^{2} / 2\right) . \eta_{2}$ is the efficiency of conversion of the gas thermal energy to kinetic energy. For an ideal infinite nozzle and zero back pressure $\eta_{2}=1$.

$$
\text { We had: } \eta_{I}=\frac{M_{p} v_{f}^{2}}{M_{f} v^{2}} \text {. }
$$

According to the rocket equation for constant exhaust velocity,

$$
v_{f}=v \ln \mu=v \ln \left(\frac{M_{s}+M_{f}}{M_{s}}\right) \text {, }
$$


this becomes simply

$$
\eta_{1}=\frac{\ln ^{2} \mu}{\mu-1} \text {. }
$$

$\mu$ is the rocket mass ratio. By inspection it is clear that $\eta_{1}$ tends to zero for very large or very small $\mu$. For $\mu=4.9$ we obtain the maximum value $\eta_{1}$ may have: $\eta_{1}=0.65$. Only by relaxing the condition that the exhaust velocity be constant may $\eta_{\text {be increased. This possibility is }}$ discussed in IV. A plot of $\eta_{1}$ vs. $\mu$ is shown in Fig. 2.

The exhaust velocity $\mathrm{v}$ and gas temperature, molecular weight, and specific heat are related by

$$
\begin{aligned}
v & =\eta_{2} \quad \frac{2 \gamma}{\gamma l} \frac{R_{0} T}{w} \\
\eta_{2} & =\left\{\begin{array}{cl}
1 & \text { steady operation, infinite nozzle } \\
\frac{2}{\gamma+1} & \text { pulsed operation (IV), infinite nozzle } \\
\sqrt{\frac{\gamma-1}{2}} & \text { steady operation, no nozzle }
\end{array}\right.
\end{aligned}
$$

III. SIGNIFICANCE OF THE THROUGHPUT REQUIREMENT

The first thing we notice about the throughput requirement is that neither the gas temperature and pressure nor absolute size of the device determine $N$. The temperature and exhaust velocity enter only into the determination of $\eta_{1}, \eta_{2}$. As for the gas pressure and device size, we have only required that the thin shell approximation for calculating the failure pressure be valid. If it is not valid, then $M_{s}$ and hence $N$ are increased. It is also interesting to observe that the molecular weight of the propellant gas does not enter into determining $N$, but only $\gamma$. Needless to say, if $W$ is large, so is the required value of $T$, resulting in greater difficulty in maintaining the structural integrity of the pressure shell material.

Although the quantity $\mathrm{N}$ is a characteristic of all systems which contain the gas during the heat addition process, it is not of uniform importance for all systems. Clearly, it is of no importance for the heat exchanger type since only one critical mass of fissionable material 
is required. For the type which throws away one critical mass per throughput, $\mathrm{N}$ is a very important parameter as may be shown by a typical example.

Let us assume that the propellant is molecular hydrogen $(\gamma=7 / 5)$, steady-state operation with an infinite nozzle $\left(\eta_{2}=1.0\right)$, and a steel pressure shell with strength $\sigma_{s}=30,000$ psia. Although the assumed steel tensile strength might seem conservative, the pressure shell probably cannot be kept cold so this represents a "warm" strength. Furthermore, in a realistic pressure shell design there are nuts, bolts, flanges, and stress concentrations. As a result, actual weights are about a factor of four higher than given by (3). Thus, a real pressure shell with $\sigma_{s}=120,000$ psia weighs the same as one calculated from (3) with $\sigma_{s}=30,000$ psia. Then, if we express $V$ in units of ()$=\frac{V_{f}}{10^{6} \mathrm{~cm} / \mathrm{sec}}$, we have

$$
N \geqslant 822 \omega^{2}
$$

It is now clear that, for missions with $\mathrm{v}_{f}>10^{6} \mathrm{~cm} / \mathrm{sec}$, reduction of the critical mass or of the fissionable material consumed per throughput is a problem of fundamental importance to those schemes which seek to attain high exhaust temperatures by direct mising of the propellant and fissionable material. Even if the critical mass $M_{c}$ is made as low as $1 \mathrm{~kg},{ }^{l}$ the situation is rather unsatisfactory in spite of the very optimistic assumption of no structural weights.

An alternate approach does exist, of course, We can examine in more detail the assumptions used for the calculation of the constant $B$ and try to decrease its value by one or two orders of magnitude. For example, in the heat exchanger type of nuclear rocket engine an appreciably smaller value (factor of 2) of $B$ already exists because of the non-uniform gas density. One might expect that by pulsed operation higher instantaneous pressures could be achieved which would more than compensate for the reduction in mean exhaust velocity. In the case of hydrogen, dissociation in the reaction chamber would increase the gas energy content by about a factor of 5 at a given pressure. As will be seen, gas temperatures in excess of $3500^{\circ} \mathrm{K}$ are necessary before this becomes a fruitful approach.

${ }^{1}$ George Safonov, The Criticality and Some Potentialities of "Cavity Reactors," Rand Corporation Report No. RM-1520, July 17, 1955. 


\section{VARIOUS MEANS FOR DECREASING B}

\section{A. Gas density variations}

Let us now consider steady-state devices and see what might be done to decrease the value of $B$. A correction should be made for non-uniform gas density in most cases so this will be looked at first.

In order to simplify the calculation, assume the pressure shell to be a short cylinder of length $\mathrm{I}$. If the nozzle throat area is small compared to cylinder cross section, the axial pressure drop should be small, and we can assume

$$
P_{\mathrm{T}}=\text { constant; } \quad P=\text { gas density }
$$

Now, calculations by $\mathrm{H}$. Reynolds at this laboratory have indicated that moderate changes in fissile material concentration in a thermal reactor do not affect the flux distribution to first order. Thus, a crude estimate of the normalized axial power distribution $g(\Sigma)$ can be made by taking

$$
\begin{aligned}
g(\Sigma)=(\text { fuel conc. })(f l u x)=\left(\frac{\rho}{K_{1}}\right) & \left(\frac{\pi}{2} \text { sin } \pi \Sigma\right) \\
& \Sigma=\frac{x}{L} \quad 0 \leqslant \Sigma \leqslant 1
\end{aligned}
$$

where we are assuming for the axial flux distribution that which is associated with a bare cylinder. $K_{1}$ is a normalization constant obtained from the condition

$$
\int_{0}^{1} g(\Sigma) d \Sigma=1
$$

If the initial and final gas temperatures and densities are $T_{1}, P_{1}$, $\mathrm{T}_{2}, P_{2}$, then the gas temperature equation is [using the condition $\left.(14)\right]$ :

$\mathrm{dT}=\left(\mathrm{T}_{2}-\mathrm{T}_{1}\right) \mathrm{g}(\Sigma) \mathrm{d} \Sigma=\left(\mathrm{T}_{2}-\mathrm{T}_{1}\right) \frac{\pi}{2} \frac{\mathrm{T}_{1}}{\mathrm{~K}_{1} \mathrm{~T}(\Sigma)} \sin \pi \Sigma \mathrm{d} \Sigma$

Integrating,

$$
\mathrm{T}^{2}(\Sigma)=\mathrm{T}_{1}^{2}+\mathrm{T}_{1}\left(\mathrm{~T}_{2}-\mathrm{T}_{1}\right) \frac{P_{1}}{\mathrm{~K}_{1}}(1-\cos \pi \Sigma)
$$

Therefore, using (14),

$$
P(\Sigma)=\frac{P_{1}}{\sqrt{1+\frac{T_{2}-T_{1}}{T_{1}} \frac{P_{1}}{K_{1}}(1-\cos \pi \leq)}}
$$


If we let

$$
\mathrm{d}=\frac{\mathrm{T}_{2}-\mathrm{T}_{1}}{\mathrm{~T}_{1}} \frac{P_{1}}{\mathrm{~K}_{1}}
$$

we find for the average gas density

$$
P=\frac{P_{1}}{\sqrt{1+d}} \int_{0}^{1} \frac{d \Sigma}{\sqrt{1-\frac{d}{1+d} \cos \pi \varepsilon}}
$$

Now, the value of this integral is unchanged by changing the minus sign to a plus sign. Thus, by making the substitutions

$$
k^{2}=\frac{2 d}{1+2 d}, \quad z=2 \pi \Sigma
$$

we obtain

$$
\begin{gathered}
\int_{0}^{1} \frac{d \Sigma}{\sqrt{1-\frac{d}{1+d} \cos \pi \Sigma}}=2 \sqrt{\frac{1+d}{1+2 d}} \int_{0}^{\frac{\pi}{2}} \frac{d z}{\sqrt{1-k^{2} \sin ^{2} z}} \\
=2 \sqrt{\frac{1+d}{1+2 d}} F\left(\frac{\pi}{2}, z\right)
\end{gathered}
$$

$F\left(\frac{\pi}{2}, z\right)$ is the complete elliptic function of the first kind.

Thus, using (13) we find

$$
\frac{P}{P_{2}}=\frac{2}{\pi} \mathrm{F}\left(\frac{\pi}{2}, \mathrm{k}\right)
$$

Now consider the normalization condition (15). We have, using (17),

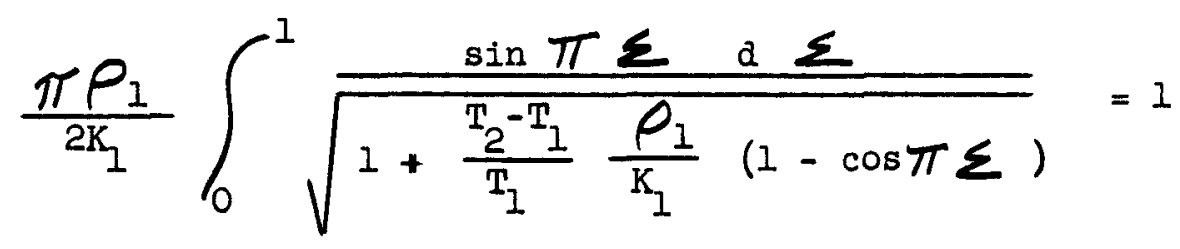

This integral may be done immediately and the result, together with a little algebra, yields

$$
\frac{T_{2}}{T_{1}}=\sqrt{1+2 d}
$$


Equations (18), (21), (23) now permit us to plot $\bar{P} / P_{2}$ vs. $\frac{\mathrm{T}_{2}}{\mathrm{~T}_{1}}$ and this is done in Fig. 3. For $\frac{T_{2}}{T_{1}}>10$ the constant pressure approximation begins to fail, and a more rigorous treat of both heat addition and neutronics is indicated. However, we see that $P$ can be about two or three times larger than $P_{2}$.

Earlier it was assumed that the gas temperature and density were uniform and equal to the exit value. We now see that the average density is somewhat higher and the required number of throughputs $N$ correspondingly reduced. Further, the higher density and lower temperature near the inlet might reduce the critical mass somewhat.

\section{B. Variable exhaust velocity}

Another assumption which was made in II was that the exit gas velocity should be constant. Let us see what happens if we drop this assumption and instead program the exhaust velocity according to the amount of propellant which has been consumed. All other assumptions are to be the same as in II. Consider a gas reactor rocket with mass ratio $\mu=\left(M_{s}+M_{f}\right) / M_{s}$ and require that its final velocity be $V_{f}$. Let $m$ be the amount of propellant which has been consumed at some instant during the thrust period. Let the exhaust velocity be a function $v(m)$. From (3), (5), (10), the mass of gas contained at any instant is

$$
M_{g}=\frac{\eta_{2}^{2} M_{s}}{B v^{2}(m)}
$$

The equation for the number of throughputs is then

$$
\mathrm{dN}=\frac{\mathrm{dm}}{\mathrm{M}_{\mathrm{g}}}=\mathrm{D \textrm {v } ^ { 2 }}(\mathrm{m}) \mathrm{dm}, \quad \mathrm{D}=\frac{\mathrm{B}}{\mathrm{M}_{\mathrm{s}} \eta_{2}^{2}}
$$

The total number of throughputs is

$$
N=D \int_{0}^{M} v^{2}(m) d m
$$

The problem is to find the functional form of $\mathrm{v}(\mathrm{m})$ which minimizes this integral subject to the auxiliary condition

$$
v_{f}=\int_{0}^{M_{f}} \frac{v(m) d m}{M_{i}-m}=\text { const., } M_{i}=M_{s}+M_{f}
$$


The solution of this variational problem is obtained by solving the Euler equation ${ }^{2}$ :

$\frac{\partial}{\partial v} \frac{v(m)}{M_{i}-m}+\lambda \frac{\partial}{\partial v} v^{2}(m)=0$

We have then,

$$
v(m)=-\frac{1}{2 \lambda\left(M_{i}-m\right)}
$$

$\lambda$ may be evaluated by substituting (29) into (27). The result for $\mathrm{v}(\mathrm{m})$ is

$v(m)=v_{f} \frac{M_{i}\left(M_{i}-M_{f}\right)}{M_{f}\left(M_{i}-m\right)}=\frac{v_{f}}{\mu-I} \frac{M_{i}}{M_{i}-m}$

Note that

$$
v_{f}=\frac{\mu-1}{\mu} v_{\max }<v_{\max }
$$

That is, although this velocity program minimizes $N$, it leads to higher exhaust velocity and temperature requirements than did the constant $v$ case. On the other hand, for $v_{f} \simeq 10^{6} \mathrm{~cm} / \mathrm{sec}$, the optimum constant exhaust velocity $v$ is about $6.3 \times 10^{5} \mathrm{~cm} / \mathrm{sec}$, which corresponds to a temperature $\mathrm{T}<2000^{\circ} \mathrm{K}$ for molecular hydrogen.

Clearly, this procedure also gives the maximum value $\eta_{I}$, the mechanical rocket efficiency, may have for a given value of $\mu$. We have for the optimally programmed case,

$$
\eta_{1}=\frac{M_{p} v^{2}}{\int_{f_{v}{ }^{2}(m) d m}^{M_{m}}}=\frac{\mu-1}{\mu}
$$

A plot of $\eta_{1}$ vs. $\mu$ is shown for both the constant exhaust velocity case and the programmed exhaust velocity case in Fig. 4. Note that this result is valid only if gravitational field effects may be ignored.

2 Richard Courant, Differential and Integral Calculus, Interscience, 1936, Vol. II, p. 520 . 
From (5), (6), (32), we now calculate the minimum number of throughputs to be

$$
\mathrm{N}=\frac{\mu}{\mu-1} \frac{\mathrm{B} \mathrm{v}^{2}}{\eta_{2}}
$$

which is at best $(\mu>>1)$ some $30 \%$ less than that given by (12).

C. Dissociation of hydrogen

Now let us turn our attention to the possibility of dissociating hydrogen gas in the reactor chamber and recovering the dissociation energy by recombination and conversion to directed kinetic energy in the nozzle. The dissociation energy is about $10^{5} \mathrm{cal} / \mathrm{mole}$ and if recovered would contribute about $10^{6} \mathrm{~cm} / \mathrm{sec}$ to the exhaust velocity.

This process is best investigated by considering the thermodynamics of the process first and then considering various limitations which arise.

We want to construct an enthalpy-entropy diagram for hydrogen in the range of temperatures and pressures of possible interest. Then the exhaust velocity is obtained by calculating the change in enthalpy for a given isentropic process.

Consider one mole of undissociated $\mathrm{H}_{2}$ at some standard reference temperature at which the dissociation energy $\Delta_{h}{ }^{\circ}$ is known. Then, at some other temperature $T$ the enthalpy is just

$$
\begin{aligned}
\mathrm{h}=(1-\mathrm{y}) \mathrm{h}_{2}+2 \mathrm{yh} & +\mathrm{y} \Delta \mathrm{H}^{\circ}+\mathrm{yh}_{2}^{\circ}-2 \mathrm{yh}_{1}^{\circ} \\
\mathrm{y} & =\text { fraction of } \mathrm{H}_{2} \text { dissociated } \\
\mathrm{h}_{2} & =\text { enthalpy of } \mathrm{H}_{2} \text { gas at } 1 \text { atmosphere, temperature } \mathrm{T} \\
\mathrm{h}_{2}^{\circ} & =\text { enthalpy of } \mathrm{H}_{2} \text { gas at } 1 \text { atmosphere, reference temp- } \\
\mathrm{h}_{1}^{\circ} & =\text { enthalpy of } \mathrm{H} \text { gas at } 1 \text { atmosphere, reference temp- } \\
& \text { erature } \\
\mathrm{h}_{1}= & \text { enthalpy of } \mathrm{H} \text { gas at } 1 \text { atmosphere }
\end{aligned}
$$

Using data ${ }^{3}$ on $\mathrm{H}_{2}$ and $\mathrm{H}$ gas this becomes

$$
\mathrm{h}=\mathrm{h}_{2}+\mathrm{y}\left(2 \mathrm{~h}_{1}-\mathrm{h}_{2}\right)+103,232 \mathrm{y} \quad \mathrm{cal} / \mathrm{mole}
$$

3 Harold J. Wooley, Russell B. Scott and F. G. Brickwedde, Compilation of the Thermal Properties of Hydrogen in its Various Isotopic and Orthopara Modifications, Journal of Research N.B.S., 41, 393, (1948) 
When $y$ moles of $\mathrm{H}_{2}$ are dissociated, the mole fractions of $\mathrm{H}_{2}$ and $\mathrm{H}$ respectively are $\frac{1-y}{1+y}, \frac{2 y}{1+y}$. The entropy $s$ can then be written

$$
s=(1-y) s_{1}+2 y s_{2}-(1+y) R_{0} \operatorname{lnp}-s_{\operatorname{mix}}
$$

where

$$
\begin{aligned}
s_{2} & =\text { entropy of } \mathrm{H}_{2} \text { gas at } 1 \text { atmosphere, temperature } \mathrm{T} \\
\mathrm{s}_{1} & =\text { entropy of } \mathrm{H} \text { gas at } 1 \text { atmosphere, temperature } \mathrm{T} \\
\mathrm{p} & =\text { pressure (atmosphere) } \\
\mathrm{s}_{\operatorname{mix}} & =\text { entropy of mixing } \\
s_{\operatorname{mix}} & =(1-\mathrm{y}) \ln \left(\frac{1-\mathrm{y}}{1+\mathrm{y}}\right)+2 \mathrm{y} \ln \left(\frac{2 \mathrm{y}}{1+\mathrm{y}}\right)
\end{aligned}
$$

Now $s_{\operatorname{mix}}<0$ and has a maximum magnitude of $0.96 \mathrm{cal} / \mathrm{mole}$ degrees. Since $s \sim 100 \mathrm{cal} / \mathrm{mole}$ degrees, $s_{\operatorname{mix}}$ is neglected here.

The fraction $\mathrm{y}$ which is dissociated at a given temperature and pressure is given by

$$
y=\sqrt{\frac{K(T)}{K(T)+4 p}}
$$

The dissociation constant $K$ may be calculated from the free energy change of the reaction, but in this report the values used at $3000^{\circ}, 4000^{\circ}$ and $5000^{\circ}$ were published values (See Ref. 3, page 14). Interpolated values may be obtained using the relation,

$$
\frac{d K}{K}=\frac{\Delta h}{R T^{2}} d T
$$

where $\Delta_{h}=\Delta_{\mathrm{h}}{ }^{\circ}+2\left(\mathrm{~h}_{1}-\mathrm{h}_{1}{ }^{\circ}\right)-\left(\mathrm{h}_{2}-\mathrm{h}_{2}{ }^{\circ}\right)$

from conservation of energy. The calculated values of $y$ together with the values of $\mathrm{K}$ used are shown in Fig. 5 .

Isotherms and isobars are plotted on the h-s diagram in Fig. 6. The gas is conceived to be initially hot and nearly dissociated, thus having a high value of $h$. The expansion process through the nozzle is represented approximately on the $h-S$ diagram by a vertical line leading to lower enthalpy values. Note that there is a corresponding large drop in pressure. The exhaust velocity is just

$$
v=\sqrt{\frac{2 \delta h}{W}} \quad \mathrm{~cm} / \mathrm{sec} \quad\left(\delta_{h}=\text { enthalpy change }\right)
$$

In this case the equation of state of the gas in the chamber is

$$
\rho=\frac{W p}{R_{0} T(I+y)}
$$


Comparing the values of $\mathrm{N}$ in this case with the value calculated on the basis of no dissociation we find that the new value of $N$ is (assume $\mu, T$ are the same in both cases):

$$
\begin{aligned}
& N^{\prime}=\frac{(1+y)}{\phi} N \\
& \text { where } \phi \text { is the ratio } \\
& \varnothing=\frac{\delta \mathrm{h}}{\mathrm{h}_{2}(\mathrm{~T})}
\end{aligned}
$$

Now $(1+y) \simeq 2, \delta h \sim \Delta h^{0}+h_{2}(T)$, so that $\phi \leq 5$ unless $T<3000^{\circ} \mathrm{K}$. For $\mathrm{T}<3000^{\circ} \mathrm{K}$, we find that the available $\delta_{\mathrm{h}}$ decreases rapidly with decreasing temperature. In the first place, the chamber pressure required to have $\mathrm{y} \sim 1$ becomes quite small ( $\sim 0.1$ atmosphere). But then the recombination rate becomes too slow to maintain equilibrium during the expansion process.

The hydrogen recombination occurs almost exclusively by a threebody collision between three hydrogen atoms. The specific reaction rate is $^{4}$ :

$$
\mathrm{k}_{3} \sim 10^{16} \frac{\mathrm{cm}^{6}}{\mathrm{~mole}^{2} \mathrm{sec}}
$$

Thus, the fraction recombining per second is:

$$
f=k_{3}\left(\frac{p}{R_{0} T}\right)^{2}
$$

For a thirty-foot-long nozzle, the transit time of the gas is $\sim 10^{-3}$ sec. Therefore, $f>10^{3}$ in order to have thermal equilibrium. Using $\mathrm{T}=3000^{\circ} \mathrm{K}$ we find that $p>7.9 \times 10^{4}$ dynes $/ \mathrm{cm}^{2} \simeq 0.10$ atmosphere.

Thus, a factor of two reduction in $\mathrm{N}^{\prime}$ is about all one can hope for in this fashion and then only for a limited range of chamber temperatures-say 4000 to $6000^{\circ} \mathrm{K}$.

\section{Pulsed operation}

Let us now turn to pulsed systems. In these we imagine that at some instant the reactor cavity is filled with gas at some pressure and

\footnotetext{
${ }^{4}$ Glasstone, Laidler \& Eyring, The Theory of Rate Processes, lst ed., McGraw-Hill, 1941, p. 219.
} 
temperature p, T. Subsequently, the gas is allowed to escape through a nozzle until the cavity is exhausted and the process is repeated. The hope is that higher temperatures and pressures may be achieved in this manner. The filling process is undetermined for the moment. We shall assume that the system is in thermodynamic equilibrium.

First consider the specific impulse of such a system. Since the process of exhausting the cavity is an adiabatic expansion, we have for the gas inside:

$$
\begin{aligned}
& \text { p } \rho^{-\gamma}=\text { constant }=K_{2} \\
& \text { or } \\
& \text { T } p^{\frac{\gamma}{\gamma-1}}=\text { constant }
\end{aligned}
$$

Assume the propellant to be a perfect gas with constant molecular weight so that

$$
p=\frac{\rho R_{0} T}{W}
$$

Since the velocity at the nozzle throat is sonic, we have, using $(44)$,

$$
G^{2}=\frac{\left(p^{*}\right)^{2} A W}{R_{0} T^{*}}
$$

where the star indicates the quantity in question is measured at the nozzle throat. G is the mass flow rate through the nozzle throat area $A$. The starred quantities are related to those within the chamber by the expressions,

$$
\begin{aligned}
& \mathrm{T}=\frac{\gamma+1}{2} \mathrm{~T}^{*} \\
& \mathrm{p}=\left(\frac{\gamma+1}{2}\right)^{\frac{\gamma}{\gamma-1} \mathrm{p}^{*}}
\end{aligned}
$$

Since we are assuming complete conversion of thermal energy to kinetic energy, the specific impulse at any moment is related to the chamber temperature by

$$
I=\frac{1}{g} \sqrt{\frac{2 \gamma}{\gamma-1} \frac{R_{0} T}{W}},
$$

where $\mathrm{g}$ is the acceleration due to gravity. 
If the cavity volume is $v$, the mass of gas contained at any time $t$ is

$$
\mathrm{M}_{\mathrm{g}}=\rho_{\mathrm{V}}=\left(\frac{\mathrm{p}}{\mathrm{K}_{2}}\right)^{\frac{1}{\gamma}}
$$

so

$$
\frac{d M_{B}}{d t}=\frac{V}{\gamma_{K_{2} / \gamma}} p^{\frac{1-\gamma}{\gamma}} \frac{d p}{d t} .
$$

Also, from (43), (44), (45),

$$
G^{2}=\frac{V\left(p^{*}\right)^{2} A^{2}}{R T^{*}}=\frac{V A^{2}}{K_{2} / \gamma}\left(\frac{\gamma+1}{2}\right)^{-\frac{\gamma+1}{\gamma-1}} \frac{\gamma^{+1}}{p^{\gamma}}
$$

But $G=-\frac{d M}{d t}$ by definition, so

$$
\sqrt{\frac{\gamma_{A}{ }^{2}}{k_{2} / \delta}\left(\frac{\gamma+1}{2}\right)^{-\frac{\gamma+1}{\gamma-1}}} p^{\frac{\gamma+1}{2 \gamma}}=-\frac{v}{\gamma_{K_{2}} 1 / \gamma} p^{\frac{1-\gamma}{\gamma}} \frac{d p}{d t}
$$

which has the solution:

$$
p(t)=p(0)\left(1+\frac{t}{\theta}\right)^{-\frac{2 \gamma}{\gamma-1}}
$$

where

$$
\theta=\frac{V}{A c_{0}}\left(\frac{2}{\gamma-1}\right)\left(\frac{\gamma+1}{2}\right)^{\frac{\gamma+1}{2(\gamma-1)}}
$$

$\mathrm{C}_{0}$ is the chamber sonic velocity at time $t=0$.

From equations (43), (47), (48), the temperature, mass of gas, and specific impulse are found to vary with time according to the equations:

$$
\begin{aligned}
& T(t)=T(0)\left(1+\frac{t}{\theta}\right)^{-2} \\
& M_{g}(t)=M_{g}(0) \quad\left(I+\frac{t}{\theta}\right)^{-\frac{2}{\gamma / I}} \\
& I(t)=I(0) \quad\left(1+\frac{t}{\theta}\right)^{-1}
\end{aligned}
$$

Equations (52) and (54) are plotted in Fig. 7. Note that $\theta$ is the 
characteristic time for decrease of the specific impulse by a factor of two. Now let's put in some numbers to obtain an idea of the times involved. Consider atomic hydrogen at $\mathrm{T}(\mathrm{O})=4000^{\circ} \mathrm{K}$, chamber radius of $25 \mathrm{~cm}$, and chamber cross-sectional area ten times that of the nozzle throat. Then, $\theta=2.54 \times 10^{-3} \mathrm{sec}$. In time $t=0.4 \theta$ about half the gas has escaped, and I has been reduced by about $20 \%$.

We can find the average specific impulse most simply by expressing $I$ as a function of the remaining gas $M_{g^{*}}$ Then:

$$
\bar{I}=\int_{0}^{M_{g}(0)} \frac{I d M_{g}}{M_{g}(0)}
$$

Using equations (43), (44), (47) and (48) we find that I is given in terms of $\mathrm{M}_{\mathrm{g}}$ by:

$$
I=\frac{v}{g} \sqrt{\frac{1-\gamma}{2}} \sqrt{\frac{2 K_{2}}{\gamma-1}} \mathrm{M}_{\mathrm{g}}^{\frac{\gamma-1}{2}}=P \mathrm{M}_{\mathrm{g}}^{\frac{\gamma-1}{2}}
$$

and

$$
\bar{I}=\frac{P}{g M_{g}(0)} \frac{2}{\gamma+1}\left[M_{g}(0)\right]^{\frac{\gamma+1}{2}}=\frac{2}{\gamma+1}^{\frac{\gamma}{\gamma}} \quad(0)
$$

Thus, pulsed operation reduces the specific impulse by a factor $\frac{2}{\gamma+1}$ for a given temperature of operation. This result is not valid if a change in the molecular weight occurs during the exhausting process. For example, one might have dissociated hydrogen initially present with recombination taking place in the chamber as the pressure and temperature fall. The temperature dependence of dissociation is exponential so this is most serious.

The penalty paid in specific impulse for pulsed operation was seen to be small and could probably be compensated in any case by increased initial temperature. Therefore, we now turn to a consideration of the pressures which might be sustained. In particular, we want to see if the initial pressure can be appreciably larger than the steady-state failure pressure without incurring failure. 
If we have a spherical shell of thickness $T_{s}$ and radius $R$ at zero pressure, then upon application of an internal gas pressure $p$ the shell tensile stress $s$ is

$$
s=\frac{p R}{2 T_{s}}
$$

The radial displacement $r$ due to this pressure is just

$$
r=\frac{S}{Y} R
$$

where $Y$ is the Young's modulus of the shell.

Combining (57) and (58) we have

$$
\mathbf{P}=\frac{2 Y T}{R^{2}} \mathbf{r} \text {. }
$$

Now assume that in steady state, failure occurs when $\sigma_{s}=s$. Therefore, the failure pressure and displacement are

$$
\begin{aligned}
& p_{f}=\frac{2 \sigma_{s} T_{s}}{R} \\
& r_{f}=\frac{R}{2 Y T_{s}} p_{f}
\end{aligned}
$$

Before considering the effect of an impulse let us first consider free radial oscillations in the fundamental mode. Since the pressure $p$ is just equal and opposite to the radial restoring force associated with the displacement $r$ (static case), we may write imediately the equation of motion,

$$
\rho_{\mathrm{s}} \ddot{x}+\frac{2 \epsilon}{R^{2}} r=0
$$

or

$$
\ddot{r}+4 \pi^{2} v^{2} r=0
$$

where

$$
4 \pi^{2} v^{2}=\frac{2 Y}{\rho_{s} R^{2}}=\frac{2\left(C^{*}\right)^{2}}{R^{2}}
$$

$V$ is the oscillation frequency and $c^{*}$ is the longitudinal sound velocity for the shell material.

Now suppose that we impose an impulse of pressure $p_{0} f(t)$ upon the static unstressed sphere $[f(0)=1]$. By an impulse we mean that $f(t)$ becomes very small in a time $t \sim 1 / v$. Then, 


$$
\ddot{r}+4 \pi^{2} v^{2} r=\frac{p_{0} f(t)}{\rho_{s} T_{s}} \quad, \quad f(t) \leq 1
$$

We may integrate (63) over the duration of an impulse, neglecting the second term, and find

$$
\dot{r}_{0}=\frac{1}{\rho_{s} T_{s}} \int_{0}^{\infty} p_{0} f(t) d t
$$

Let us define the length of the impulse $\tau$ by

$$
p_{0} \tau=\int_{0}^{\infty} p_{0} f(t) d t
$$

The amplitude a of the resulting free oscillation is just

$$
2 \pi a=\frac{\dot{r}_{0}}{\nu}=\frac{p_{0} T}{v P_{s} T_{s}}
$$

We assume that failure occurs if $a>r_{f}$. If $a=r_{f}$ then

$$
\frac{p_{0}}{2 \pi \nu P_{s} T_{s}}=\frac{R^{2}}{2 Y T_{s}} p_{f}
$$

so that

$$
\frac{p_{0}}{p_{f}}=\frac{1}{2 \pi \sqrt{T}}
$$

Since $v \mathbb{T}<1$, we see that the impulsive pressure $p_{0}$ may be quite large compared to $p_{f}$ before failure occurs.

This situation may be improved further by a factor of 2 if the sphere is already oscillating with an amplitude $a \simeq r_{f}$. Then, if the impulse is applied when

$$
r=0, \quad \dot{r}=-\frac{a}{2 \pi \nu},
$$

it may be of magnitude such that $\dot{r}_{0}=\frac{a}{2 \pi \nu}$, thereby merely changing the phase of the oscillation by $\pi$. Then

$$
\frac{p_{0}}{p_{f}}=\frac{2}{2 \pi r \tau}
$$


Unfortunately, we can now show that the impulse approximation is not likely to be satisfied. For the pressure impulse we had from (52),

$$
p(t)=p_{0}\left(\frac{1+t}{\theta}\right)^{-\frac{2 \gamma}{\gamma-1}}
$$

Then,

$$
p_{0} \tau=p_{0} \int_{0}^{\infty}\left(1+\frac{t}{\theta}\right)^{-\frac{2 \gamma}{\gamma-1}} d t=p_{0} \theta \frac{\gamma-1}{\gamma+1}
$$

and

$$
\tau=\frac{\gamma-1}{\gamma+1} \theta
$$

From (53, (62), (69), we now find

$$
2 \pi \nu \tau=\frac{4 \sqrt{2} c^{*}}{3 c_{0}}\left(\frac{R}{x}\right)^{2}\left(\frac{\gamma+1}{2}\right)^{\frac{3-\gamma}{2 \gamma-1}}
$$

$X$ is the radius of the nozzle throat.

By definition, $R>X$. For steel, $C^{*} \simeq 5 \times 10^{5} \mathrm{~cm} / \mathrm{sec}$, while for $\mathrm{H}_{2}$ at $3000^{\circ} \mathrm{K}, \mathrm{C}_{\mathrm{O}}=4 \times 10^{5} \mathrm{~cm} / \mathrm{sec}$. For $\gamma=1.40$ the remaining factor is 2.71 .

If we look at materials other than steel we find that a reduction in the sound velocity $\mathrm{C}^{*}$ is accompanied by a much larger reduction in the tensile strength. Further, in order to realize the calculated specific impulse for a pulsed system we should probably require that $x^{2}<<R^{2}$. The result of all this is that in practice $\nu Y>>1$ except for very high $\left(>50,000^{\circ} \mathrm{K}\right)$ gas temperatures.

Since we are likely to find $\gamma \Psi>>1$, we may estimate the failure pressure by considering the pressure function to be a step function rather than an impulse. The equation of motion is then

$$
\ddot{r}+4 \pi^{2} v^{2} r=\frac{p_{o}}{\rho_{s} T_{s}}=4 \pi^{2} v^{2} r_{f} \frac{p_{o}}{p_{f}}
$$


The general solution is

$r(t)=A_{1} \sin 2 \pi \nu t+A_{2} \cos 2 \pi \nu t+r_{f} \frac{p_{0}}{p_{f}}$

If the shell is initially static and unstressed, the boundary conditions give

$$
r(t)=r_{f} \frac{p_{0}}{p_{f}}(1-\cos 2 \pi v t)
$$

Therefore,

$$
\frac{p_{0}}{p_{f}} \leq 1 / 2
$$

If the shell is initially oscillating with amplitude a the boundary conditions may be written

$$
\begin{aligned}
& r(0)=a \sin \psi \\
& \dot{r}(0)=2 \pi v a \cos \psi
\end{aligned}
$$

$\psi$ is the phase angle at time $t=0$.

The solution is now

$$
r(t)=a \cos \psi \sin 2 \pi v t+\left(a \sin \psi-r \frac{p_{0}}{p_{f}}\right) \cos 2 \pi v t+r_{f} \frac{p_{o}}{p_{f}}(76)
$$

If $a=r_{f}$ it can be shown that the minimum value of the amplitude $a$ of the resulting forced oscillations is obtained when $\psi=\pi / 2$ and is given by

$$
\frac{a}{r_{f}}=2 \frac{p_{0}}{p_{f}}-1
$$

Since the failure condition is $\frac{a}{r_{f}} \leq 1$, we find that $\frac{p_{0}}{p_{f}} \leq 1 .(78)$

Smaller values of a lead to limits on $\frac{p_{o}}{p_{f}}$ intermediate between those
of $(74)$ and $(78)$.

We now see that the only parameter likely to be improved by pulsed operation is the gas temperature and hence the specific impulse. Possibly quite sizable increases in temperature could be realized if ceramic coatings of some type were used which would melt during the initial temperature spike and recrystallize between pulses. 


\section{INCLUSION OF PAYLOAD AND STRUCTURE}

Up to this point the discussion has been centered about minimal devices consisting only of a pressure shell, propellant, and weightless nozzles. In order to inject a more realistic note into the discussion we should now consider the effects of payload, structure and tanks upon the foregoing results.

For purposes of calculation we shall express payload plus structure weights (excluding tanks) in terms of the pressure shell weight and express the tank weight in terms of the propellant weight. Thus,

$$
\begin{aligned}
& M_{p}=\alpha M_{s} \\
& M_{t}=\beta M_{f} \\
& M_{i}=(1+\alpha) M_{s}+(1+\beta) M_{f} \quad \quad M_{i}=\text { initial mass }
\end{aligned}
$$

Then, the mass ratio is

$$
\mu=1+\frac{M_{f}}{(1+\alpha) M_{s}+\beta M_{f}}
$$

If we assume optimum velocity programing, we have from (30),

$$
v(m)=v_{f} \frac{M_{i}\left(M_{i}-M_{f}\right)}{M_{f}\left(M_{i}-m\right)} . \text { Again, } v_{f}=\frac{\mu-1}{\mu} v_{\max }
$$

This is now substituted into (26) to yield

$$
N=\frac{B v_{f}^{2}}{\eta_{2}} \frac{M_{i}\left(M_{i}-M_{f}\right)}{M_{s} M_{f}}
$$

Using (79) and (80), this can be written in terms of $\alpha, \beta, \mu$ :

$$
N=\frac{B v_{f}^{2}}{\eta_{2}} \frac{(1+\alpha) \mu}{(\mu-1)[1-\beta(\mu-1)]}
$$

Setting $\frac{\partial N}{\partial \mu}=0$ and solving for $\mu$,

$$
\mu=\mu_{0}=\sqrt{\frac{1+\beta}{\beta}}
$$


Substituting into (82) we find that the minimum value of $N$ is expressed in terms of $\alpha, \beta$, by

$$
N=(1+\alpha)(1+2 \beta+2 \sqrt{\beta(1+\beta)}) \frac{B V_{f}^{2}}{\eta_{2}}
$$

This reduces to $(33)$ as $\alpha, \beta$ tend toward zero.

Some applications of $(84)$ and (82) immediately come to mind. We may inquire first just what effect $\alpha, \beta$ have upon the minimum throughput requirements for reasonable configurations. It is unlikely that tanks can be built which have tankage factors less than about $\beta=0.04$ for hydrogen. This results from the low density of hydrogen, acceleration loads upon the system, and pump inlet requirements which appear to demand an internal pressure of about one atmosphere. If we accept this limitation, the term involving $\beta$ is found to have a value 1.5 or larger. For reasonable mission economy, the ratio $N / \alpha$ which gives throughputs per unit payload for a given value of $\mathrm{M}_{S}$ is the interesting quantity. Clearly we should have $\alpha \simeq 1$ or larger. Combining the two results, we find that the minimum value of $\mathrm{N}$ has been increased by a factor of three or more through inclusion of structural factors. Actually, neutron reflector, pump, turbine and nozzle weights are included in $\alpha$ as well as payload so that probably $\alpha \sim 4$ for good mission economy. Thus, we have more realistically an increase of $\mathrm{N}$ by about a factor of seven for a real system. This increase overrides the reductions we have been able to achieve through considerations of density variations and dissociationrecombination effects.

There is now a second point. The original reason for considering gas reactors with molecular heat transfer was that we hoped to achieve much higher exhaust temperatures (or gas velocity) and hence lower mass ratios than with a conventional system. We can still assume that this is possible and see just what the price is in terms of throughout requirements. Using the considerations of the last paragraph, let us assume $\alpha=4, \beta=.04$. Then, from (83), the optimum value of $\mu$ is $\mu=5.1$. This gives for the minimum of throughputs,

$$
N \simeq 6 \frac{v_{f}^{2}}{\eta_{2}}
$$


Now reduce $\mu$ to the low value $\mu=1.5$. Then we find that

$$
\mathrm{N} \simeq 15 \frac{\mathrm{B}^{2}}{\eta_{2}}
$$

In this case, the price has been a 2.5-fold increase in throughput requirements. Needless to say, the importance of this increase in $\mathrm{N}$ and hence total outlay of fissionable material depends upon the ratio of the cost of this material to that of the entire vehicle including payload. This will be considered further in Section VIII.

\section{MASS EFFICIENCY}

Up to this point we have been investigating the minimum throughput requirements. We have done this since, together with the existence of a minimum realizable critical mass, this requirement gives the minimum amount of fissionable material needed for a given mission requirement $V_{f}$ regardless of other considerations. Now, it may be that a nuclear fuel requirement of say $1000 \mathrm{~kg}$ or more would not be too frightening for some missions of sufficient importance which could not be carried out in any other more economical way. For example, one might find that such a large fuel expenditure resulted in a correspondingly large payload. We should therefore examine the problem from a somewhat different point of view and investigate the problem of "mass efficiency."

For the purposes of this report we may define the "mass efficiency" by the ratio of payload to fissionable material required. Thus,

$$
Q_{m}=\frac{M_{p}}{M_{\text {fiss }}}
$$

The best existing critical mass calculations on systems of the type considered here are probably those of Safonov (see Ref. 1). Extending his calculations to large $R$ for the fuel-reflector combination yielding lowest $\mathrm{M}_{\mathrm{c}}\left(\mathrm{Pu}{ }^{239}, \mathrm{D}_{2} \mathrm{O}\right)$, we find the approximate relation

$$
\mathrm{M}_{\mathrm{c}} \simeq 6 \times 10^{-2} \mathrm{R}^{2} \mathrm{gm} \quad, \quad \mathrm{R} \geqslant 100 \mathrm{~cm}
$$

At small $R$ this expression yields values of $M_{c}$ which are much too small, the error being a factor of two at $R=100 \mathrm{~cm}$. Safonov finds that 
the minimum in the critical mass occurs at $R=36 \mathrm{~cm}, M_{c}=600 \mathrm{gm}$. In his calculations, only fissionable material is assumed to exist in the core, and the reflector (which serves also as moderator) is assumed to be of infinite extent.

In the case under consideration here the atom ratio of propellant (hydrogen) to fissionable material is rather large so that the effects of moderation and parasitic capture of neutrons in the core must be considered. In addition, the reflector must be finite. The result is that a good critical mass calculation would be very difficult to carry out. One may perhaps avoid such a calculation by considering the upper limit set on the propellant to fissionable material atom ratio by parasitic capture. Regardless of shape or size of the core or reflector the nuclear chain reaction cannot be maintained unless the core propellant-to-fissionable material atom ratio satisfies the relation

$$
\frac{n_{f}}{n_{f i s s}} \leqslant \frac{M_{f i s s}}{M_{f}}\left(\eta_{f i s s}-1\right)
$$

Here

$$
\begin{aligned}
& \sigma_{\text {fiss }}=\begin{array}{l}
\text { neutron absorption (capture }+ \text { fission) cross section } \\
\text { of fissionable material }
\end{array} \\
& \sigma_{f}=\text { neutron capture cross section of propellant } \\
& \eta_{\text {fiss }}=\text { neutrons emitted per absorption by the fissionable }
\end{aligned}
$$

For thermal energies and hydrogen propellant, the maximum value of the right side of (89) is obtained with $\mathrm{Pu}^{239}$ as the fissionable material and a neutron temperature of $\sim 2000^{\circ} \mathrm{K}$. A Maxwellian velocity distribution is assumed for the neutrons. Then,

$$
\text { - } \frac{n_{f}}{n_{\text {fiss }}} \leqslant 9000
$$

The equality sign holds only for very large core size and consequently values of $M_{c}$ considerably larger (factor of 10 - 100) than the minimum value of about $0.60 \mathrm{~kg}$. 
Thus,

$$
\frac{M_{g}}{M_{c}}=\frac{W_{f}}{W_{\text {fiss }}} \cdot \frac{n_{f}}{n_{\text {fiss }}} \leqslant \frac{9000}{239}=37.6
$$

From (79),

$$
\frac{M_{p}}{M_{f}}=\frac{\alpha}{1+\alpha}\left\{\frac{1-\beta(\mu-1)}{\mu-1}\right\}
$$

And so

$$
Q_{m}=\frac{M_{p}}{M_{f i s s}} \equiv \frac{M_{p}}{M_{f}} \cdot \frac{M_{f}}{M_{f i s s}} \equiv \frac{M_{p}}{M_{f}} \cdot \frac{N M_{g}}{N M_{c}}<\frac{37.6 \alpha}{1+\alpha}\left\{\frac{1-\beta(\mu-1)}{\mu-1}\right]
$$

Now,

$$
\frac{\alpha}{1+\alpha}\{1-\beta(\mu-1)\} \leqslant 1
$$

So

$$
Q_{m} \leqslant \frac{37.6}{\mu-1}
$$

From extensive calculations which have been carried out at this laboratory and elsewhere it appears for $\omega=0.80$ and values of $M_{c}$ from 50 - $100 \mathrm{~kg}$ that heat-exchanger-type nuclear hydrogen rockets could be built with payloads of 10,000 to $100,000 \mathrm{~kg}$, yielding $\mathrm{Q}_{\text {m }}$ values of 200 1000. From (92) we see that in order to have $Q_{m}>200$ we must have $\mu<1.16$. But now we note from (5) and (9) that this increases $N$ by a factor of about 5 . We obtained this value of $Q_{m}$ by invoking the equality sign in (92) and hence are implicitly assuming that $M_{c} \sim 10-100 \mathrm{~kg}$. With $N \simeq 5 \times 525=2625$ (see Eq. 12) this implies $M_{\text {fiss }} \sim 100,000 \mathrm{~kg}$ and $M_{p} \sim 20,000$ tons. If a reasonable size core is used so that $M_{c} \sim 5 \mathrm{~kg}$, then $Q_{m}$ is reduced by a factor of 2 or so and we require $\mu<1.08$ or $N>5000$ for $Q_{m}>200$. Thus, the total fissionable material requirement remains extreme. The heat exchanger appears to be the preferred system.

We might now consider the situation for $\omega>1$ (high terminal velocity mission). For the gas reactor (one-stage) $Q_{m}$ is independent of $w$ (but $M_{\text {fiss }} \sim N M_{c} \sim \omega^{2}$ ). For the heat-exchanger rocket the situation is a little more complex. The first stage must boost to a velocity 
$\omega_{>} 0.80$ in the presence of a $1 \mathrm{~g}$ field. It appears unlikely that the payload of this stage can exceed 0.30 of the dry weight. The subsequent stages could operate at much reduced thrust (factor of 100) and thus have much lower structural weight requirements. In fact, the principal weight contributions would come from the payload itself and the tanks. For this stage we may therefore assume $\alpha>>$. Nearly all the fissionable material required is to be found in the first stage. Assuming $Q_{m}=1,000$ for this stage and boosting to $W=0.80$ we find that $Q_{m}$ for the entire heat-exchanger system is given approximately by

$$
Q_{m}^{h}=2700 e^{-1.25}
$$

For the hydrogen gas reactor,

$$
Q_{m}<\frac{37.6}{\mu-1}
$$

Assume that $M_{c}$ can be as small as $1 \mathrm{~kg}$. Then $M_{\text {fiss }}=N \mathrm{~kg} \sim \frac{822 \omega^{2}}{\mu-I}$ for $\mu \simeq 1$. If we now require that $M_{\text {fiss }} \leqslant 10,000 \mathrm{~kg}$ for the gas reactor, then

$$
Q_{m}<\frac{222}{w^{2}}
$$

and $Q_{m}>Q_{m}^{h}$ only if $\omega>4.4$. This considerably exceeds the velocity increment required for escape from the solar system at the earth's radius $(\omega \sim 2)$. In general then, hydrogen propellant gas reactors do not appear to compete with the hydrogen heat-exchanger nuclear rocket for reasonable missions.

\section{BURNUP EFFICIENCY}

We may now consider the problem briefly from a third viewpoint. So far no mention has been made of the burnup efficiency for the nuclear fuel. We may compute this quantity readily and relate it to the pressure shell mass and the critical mass or size of the device. In terms of the energy $\mathrm{E}_{\mathrm{g}}$ contained in the gas, the shell weight is just

$$
M_{S}=3 / 2(\gamma-1) \frac{\rho_{s}}{\sigma_{s}} E_{g}
$$


This assumes that the shell approximation is valid whether by the use of one thin shell or $\mathrm{n}$ thin, hydraulically coupled shells. If a fraction $Q$ of the critical mass is fissioned during one throughput then

$$
E_{g}=Q \times 0.41 \times 10^{20} \frac{\mathrm{erg}}{\text { kiloton }} \times 1.7 \times 10^{-3} \frac{\mathrm{kiloton}}{\mathrm{gm}} \times \mathrm{M}_{\mathrm{c}}
$$

and

$$
\begin{aligned}
& M_{s}=3 / 2(\gamma-1) \frac{\rho_{s}}{\sigma s} \times 7.0 \times 10^{17} Q M_{c} . \\
& \text { With } \gamma=1.4, P_{s}=7.8, \sigma s=30,000 \text { psia, this becomes } \\
& M_{s}=1.64 \times 10^{9} Q_{c} .
\end{aligned}
$$

Since $M_{c} \sim 10^{3}$ gm or more,

$$
Q<0.61 \times 10^{-9} \mathrm{M}_{\mathrm{S}}
$$

Therefore, $M_{S}>610$ tons if we are to have $Q>10^{-3}$. With the heatexchanger rocket described earlier, $M_{S} \simeq 1$ ton and $Q \simeq 10^{-3}$. For larger devices $\left(M_{c}>10^{3} \mathrm{gm}\right)$ we use the relation (88) and find

$$
Q<10^{-6} \frac{M_{S}}{R^{2}} . \quad(R>100 \mathrm{~cm})
$$

Clearly, if $Q$ is to be $>10^{-3}$, we must have $M_{S}>1.64 \times 10^{3}$ tons. By comparison, a heat-exchanger rocket with $M_{p} \sim 10$ tons, $M_{s} \sim 1$ ton, has $Q \simeq 2.0 \times 10^{-3}$.

\section{DOLLAR ECONOMY}

Actually, $Q_{m}$ does not determine the cost of performing a mission since structure, launching facilities, etc, also cost money. For purposes of simplicity, let us consider only those costs tied up in structure or fissionable material and carry out a crude cost comparison for the gas reactor rocket and heat-exchanger rocket. Structure costs will be taken to be those typical of mass-produced high-performance aircraft or about $\$ 100 / \mathrm{kg}$. For the fissionable material we may reasonably assume a cost of $\$ 16,000 / \mathrm{kg}$. 
We now assume that a reasonable parameter for comparison is the cost in dollars per $\mathrm{kg}$ of payload. Then, we define this parameter

$$
L=16,000 \frac{M_{\text {fiss }}}{M_{p}}+100 \frac{M_{s}}{M_{p}}=\frac{16,000}{Q_{m}}+\frac{100}{\alpha}
$$

For the gas reactor system (92) gives

$$
\mathrm{L}>425(\mu-1)+\frac{100}{\alpha} \text {. }
$$

It is now clear that to reduce the delivery price per unit payload we need $\alpha>1$ (as already pointed out) and $(\mu-I$ ) small. It is equally clear from the results of section $V$ that $(\mu-1)<<1$ implies $N$ large. Also from Section VI we see that (94) is optimistic and implies $M_{c}>1 \mathrm{~kg}$. Thus, reduced $L$ implies a mission for which $M_{\text {fiss }} \sim 10,000 \mathrm{~kg}$ and a cost of the order of $\$ 160$ million.

Now consider the heat-exchanger rocket. For $\omega \sim 0.80$ we have $Q \sim 1,000, \alpha \sim 0.3$, and

$$
\mathrm{L} \sim \frac{16,000}{1,000}+\frac{100}{0.3} \sim \$ 316 / \mathrm{kg} .
$$

Note that structure accounted for most of the cost here. We see from (99) and (100) that on a dollar/kg of payload basis there is really not much difference. The principal difference (assuming that both could be built) lies in the fact that the total cost for the gas reactor rocket mission, with an $\mathrm{L}$ value of $\sim \$ 300 / \mathrm{kg}$, cannot be brought below about $\$ 100$ million, which corresponds to payloads of $\sim 300$ tons.

Suppose one grants that such payloads are desirable. Then one must

consider the development and flight testing problem. At $\$ 10^{8}$ per test vehicle such a program seems completely impractical.

\section{CENTRIFUGAL SEPARATION}

It has become clear that any gas reactor rocket concept which consumes one critical mass per throughput appears doomed on economic grounds.

One scheme which has been proposed for conserving uranium in a gas reactor is the separation of the heavy and light gases by means of a centrifugal field. A technique for establishing such a field is the use of tangential injection of the gas at the outer boundary of a cylindrical 
chamber. The gas leaves the chamber through a sonic orifice on the cylinder axis. Experiments ${ }^{5}$ have shown that near sonic tangential velocities may be achieved.

Chapman and Cowling ${ }^{6}$ give an expression for the relative diffusion velocity of two non-uniform gases. It is

$$
\bar{c}_{1}-\bar{c}_{2}=\frac{n^{2}}{n_{1} n_{2}} D_{12}\left\{\frac{\partial^{n_{10}}}{\partial r}+\frac{n_{1} n_{2}\left(m_{2}-m_{1}\right)}{n \rho} \frac{\partial \ln p}{\partial r}-\frac{\rho_{1} \rho_{2}}{p \rho}\left(F_{1}-F_{2}\right)+\frac{k_{T}}{T} \frac{\partial T}{\partial r}\right\}(101)
$$

Here we shall call $\mathrm{n}_{1}$ the number $\mathrm{cm}^{3}$ of the heavy gas (uranium) and $\mathrm{n}_{2}$ the number $\mathrm{cm}^{3}$ of propellant (hydrogen). n is the total particle density, $n_{10}=\frac{n_{1}}{n}$, and $D_{12}$ is the mutual diffusion coefficient.

First let us assume $\frac{\partial T}{\partial r}=0 . F_{1}$ and $F_{2}$ are the external forces per unit mass. Here, the only external forces are gravitational and inertial (centrifugal). Hence, $F_{1}=F_{2}$. Thus only the first two terms concern us. Note that it is not the centrifugal field directly, but rather the pressure (or collision density) gradient resulting from (or causing) the centrifugal field which gives rise to separation of the components. 6

If there were no radial mass flow (for example, one may develop the centrifugal field by rapid rotation of the cylinder), then in steady state $C_{1}-C_{2}=0$. We see then that each of the two terms gives rise to a realtive diffusion velocity but in opposite directions. The term $\frac{\partial n}{\partial r}$ causes diffusion in the direction $\underline{-r}(\underline{r}=$ radius vector $)$; the term $\frac{\partial \ln p}{\partial r}$ causes diffusion in the direction $+\underline{r}$.

Now, in our case, we do have radial flow, of propellant at least, so that the steady-state requirement for separation is that $C_{1}-C_{2}+v_{r}=0$. $v=$ radial velocity and is along $-\underline{r}$. We see that a necessary (but not sufficient) condition is that the component of the diffusion velocity resulting from the $\frac{\partial \ln p}{\partial r}$ term must exceed $v_{r}$ in magnitude. Otherwise, of necessity, $\bar{c}_{1}-\bar{c}_{2}+v_{r}<0$ and no separation ensues during the time required for the gas to traverse the chamber. Therefore, a necessary condition for separation is: $n\left(m_{1}-m_{2}\right) D_{12} \frac{\partial \ln p}{\partial r}>v_{r}$.

${ }^{5}$ J.P. Hartnett and E.R.G. Eckert, Experimental Study of the Velocity and Temperature Distribution in a High Velocity Vortex Type Flow, Heat Transfer and Fluid Mechanics Institute, Stanford University Press, 1956, pp 135-145.

${ }^{6}$ Chapman \& Cowling, Mathematical Theory of Non-Uniform Gases, Cambridge, 2nd ed., 1953, p. 244. 
From conservation of momentum,

$$
\frac{\partial p}{\partial r}=\rho \frac{v_{\Theta}^{2}}{r} \quad v_{\theta}=\text { tangential velocity }
$$

So,

$$
\frac{\partial \ln p}{\partial r}=\frac{1}{p} \frac{\partial p}{\partial r}=\frac{1}{\rho_{R T}} \cdot \frac{\rho v_{\theta}^{2}}{r}=\frac{\gamma}{c_{0}^{2}} \frac{v_{\theta}^{2}}{r}
$$

Also,

$$
\begin{gathered}
\frac{\rho}{n}=\frac{n_{1} m_{1}+n_{2} m_{2}}{n}=\bar{m}=\text { average particle mass } \\
m_{1}>\bar{m}>m_{2}
\end{gathered}
$$

Let us assume that the nozzle throat area is related to the cylinder cross section by

$$
\mathbb{A}_{\mathrm{n}}=\boldsymbol{\rho}_{\mathrm{cyl}}
$$

Since we are concerned with using this device for rocket propulsion, we may assume that the exit nozzle is sonic. Also, the inlet orifices should be sonic in order to maximize $v_{\theta}$. Then, it may be shown that on the average

$$
\mathrm{v}_{\mathrm{r}}=\mathcal{\rho} \frac{2 \mathrm{R}}{\mathrm{L}} \mathrm{v}_{\theta} \quad \begin{aligned}
& \mathrm{R}=\text { cylinder radius } \\
& \mathrm{L}=\text { cylinder length }
\end{aligned}
$$

This is seen most readily by assuming the inlet orifices to be rectangular slits of length $L$.

We can now substitute (103), (104), (105), (106), (107), into (102) to obtain

$$
\gamma \frac{\left(m_{1}-m_{2}\right)}{\bar{m}} D_{12} \frac{v_{\theta}^{2}}{c_{0}^{2}}>2 \zeta \frac{R}{L} v_{\theta}
$$

or (since $r \sim R$ in region of maximum $n_{1}$ )

$$
\frac{\mathscr{I}_{\mathrm{R}}^{2}}{\mathrm{~L}}<\frac{\gamma^{\left(\mathrm{m}_{1}-\mathrm{m}_{2}\right)}}{\mathrm{m}} \mathrm{D}_{12} \frac{\mathrm{M}_{\theta}}{2 \mathrm{C}_{0}}
$$


If this is to be a rocket, we also require that the thrust-toweight ratio $\mathrm{T} / \mathrm{w}_{\mathrm{S}}>1$.

For the thrust we have

$$
\mathrm{T} \simeq \mathrm{p} \varphi_{\pi} \mathrm{R}^{2}
$$

For the cylinder weight (not mass)

$$
w_{s}>\pi \frac{\rho_{s}}{\sigma_{s}} \mathrm{p} \mathrm{L} \mathrm{R}^{2} \mathrm{~g} \quad \mathrm{~g}=980 \mathrm{~cm} / \mathrm{sec}^{2}
$$

So, $\quad \mathrm{T} / \mathrm{w}_{\mathrm{s}} \simeq \frac{\mathrm{p} \varphi_{\pi \mathrm{R}^{2}}}{\pi \frac{\rho_{\mathrm{s}} \mathrm{pLR}^{2} \mathrm{~g}}{\sigma_{\mathrm{s}}}}=\left(\frac{\varphi_{\mathrm{R}}{ }^{2}}{\mathrm{~L}}\right) \frac{\sigma_{\mathrm{s}}}{\bigodot_{\mathrm{s} \mathrm{g} \mathrm{R}} \mathrm{R}^{2}}>1$

is an additional requirement. From (109) we see that separation could be achieved merely by making $\frac{\varphi_{\mathrm{R}}^{2}}{\mathrm{I}}$ sufficiently small. (110) sets a lower limit on this quantity.

Combining,

$$
\frac{\rho_{s} g R^{2}}{\sigma_{s}}<\frac{\varphi_{R}{ }^{2}}{L}<\frac{\gamma^{\left(m_{1}-m_{2}\right)}}{\bar{m}} D_{12} \frac{M_{\theta}}{2 c_{0}}
$$

or

$$
\mathrm{R}^{2}<\frac{\sigma_{\mathrm{s}} \partial^{\left(\mathrm{m}_{1}-m_{2}\right) D_{12} \mathrm{M}_{\theta}}}{2 \rho_{\mathrm{s}} \mathrm{g} \text { III } \mathrm{c}_{0}}
$$

At room temperature, $D_{12} \sim 1, \quad M_{\theta}<1, \quad C_{0} \sim 2 \times 10^{5} \mathrm{~cm} / \mathrm{sec}$. We may choose a good steel with $\sigma_{\mathrm{s}}=120,000 \mathrm{psia}, \rho_{\mathrm{s}}=8 \mathrm{gm} / \mathrm{cm}^{2}$. Then, $\mathrm{R}^{2}<3.5\left(\frac{\mathrm{m}_{1}-\mathrm{m}_{2}}{\overline{\mathrm{m}}}\right) \mathrm{cm}$. The maximum value of $\frac{\mathrm{n}_{1}-\mathrm{m}_{2}}{\overline{\bar{m}}}$ is 239 ( $\mathrm{Pu}^{239}$ and dissociated H). Then $R<28 \mathrm{~cm}$. This size could be made critical with an infinite $\mathrm{D}_{2} \mathrm{O}$ reflector (See Ref. 1, p. 9). We have included only the pressure shell weight in (110), however. If $\mathrm{D}_{2} \mathrm{O}$ is included also (not to mention pumps, payload, propellant) the situation becomes hopeless. 
At higher temperatures, which was the initial goal, the situation is unchanged since

$$
\mathrm{D}_{12} \sim \sqrt{\mathrm{T}} \sim \mathrm{C}_{0}
$$

This transport limitation is rather general since the light propellant must pass through the heavy element region in order to be heated. It would appear that one must somehow supply external forces to the molecules, perhaps with electric or magnetic fields, in order to overcome this difficulty. In the latter case one must examine critically the weight of electrical equipment required. The reason is that typically one finds that the power/weight ratio for the best electrical power generating equipment one knows how to build today does not exceed $1 / 3 \mathrm{kw} / \mathrm{kg}$. On the other hand the relation between the thrust, exhaust gas velocity, and exhaust gas power is

$$
T h=\frac{2 P_{e}}{V}
$$

Consequently, if the electrical equipment delivers a fraction $f$ of the total energy given to the gas in order to achieve separation, there is an upper limit on the acceleration given by

$$
Q<\frac{2 \times 10^{4}}{3 f \mathrm{v}} \mathrm{g}^{\prime} \mathrm{s} . \quad\left(1 \mathrm{~g}=10^{3} \mathrm{~cm} / \mathrm{sec}^{2}\right)
$$

If we wish to have $Q>1$, then

$$
\mathrm{v}<\frac{2 \times 10^{4}}{3 \mathrm{f}} \mathrm{cm} / \mathrm{sec}
$$

Therefore, unless $f<10^{-2}$ a severe penalty in acceleration must be paid for the use of such a technique. 


\section{CONCLUSION}

Let us now try to summarize the situation. We started out by considering the throughput limitation. This is important because the device under consideration consumes one critical mass of fissionable material per throughput, and it turned out that the required number of throughputs was quite large.

We found that this limitation was set essentially by the structural properties of the material comprising the containment vessel and mission kinetic energy requirements. When the further restriction that the system be chain reacting was imposed it was found that the gaseous nuclear reactor rocket could not compete economically with the hydrogen heat transfer nuclear rocket except for extremely ambitious missions involving enormous payloads or very high terminal velocities. For the latter case $(\omega>>1)$ it could compete only if it could do the job in one stage. This, however, implies very high exhaust velocities and hence extreme chamber temperatures. Then the problem of heat transfer to the chamber walls is very severe and almost certainly could not be solved by regenerative cooling as it is in some chemical rockets. The conclusion is thus reached that the possibility of constructing an economic high specific impulse nuclear rocket utilizing gaseous heat transfer appears to be remote. 


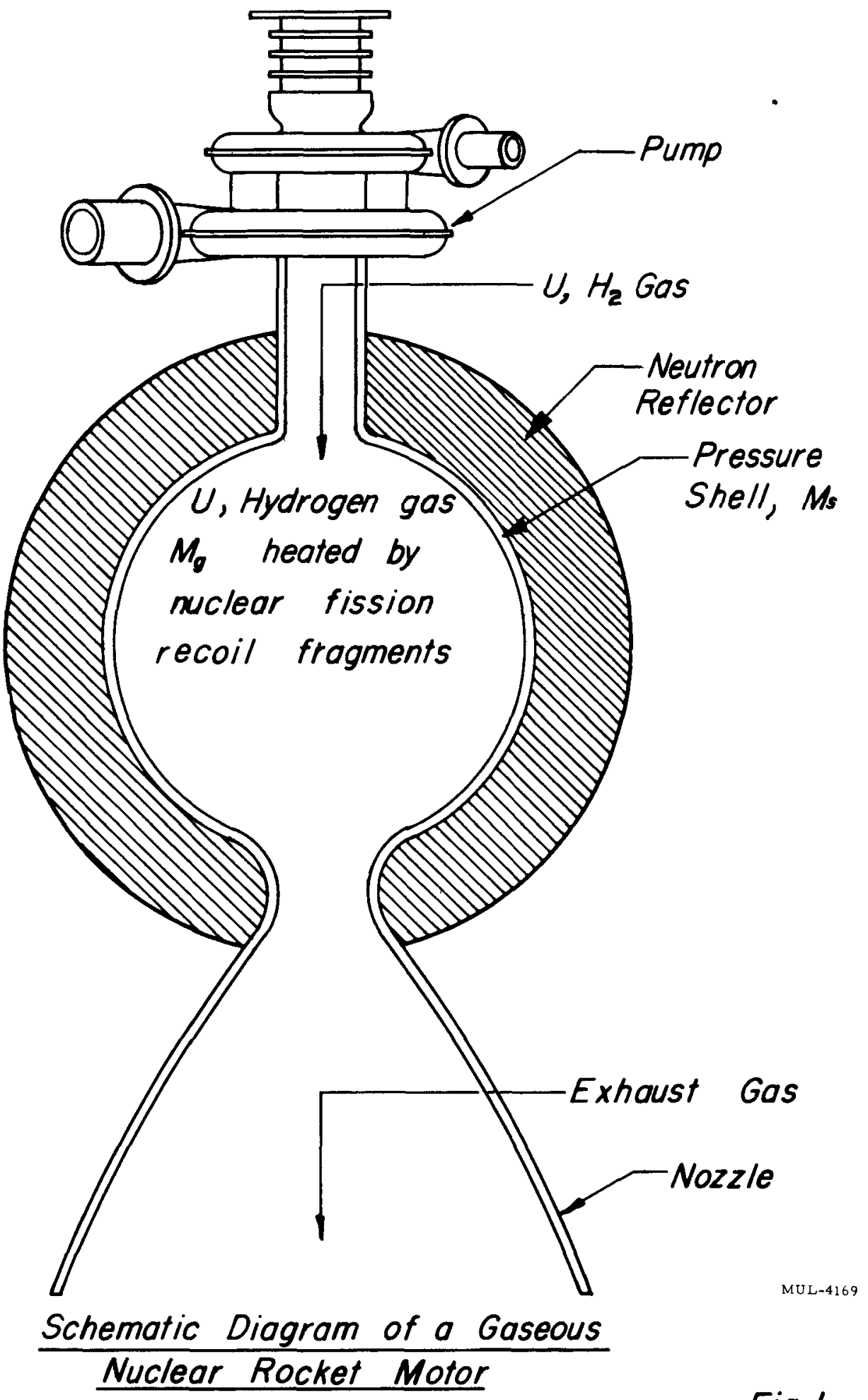

Fig. I 


\section{0 \\ $\eta$}

(2) H

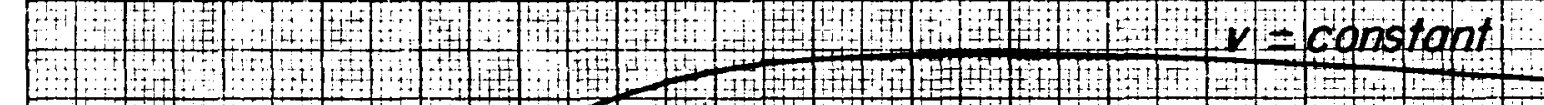

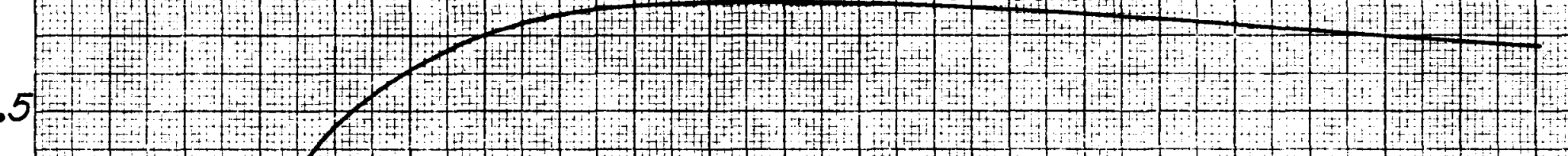

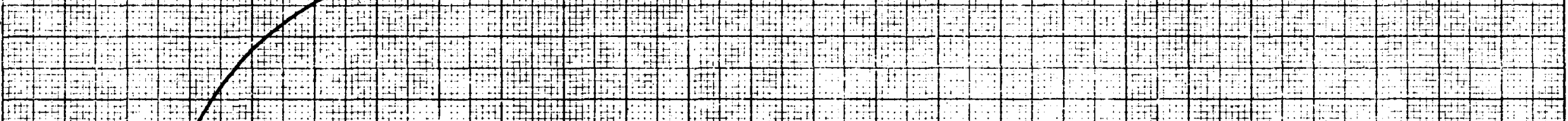

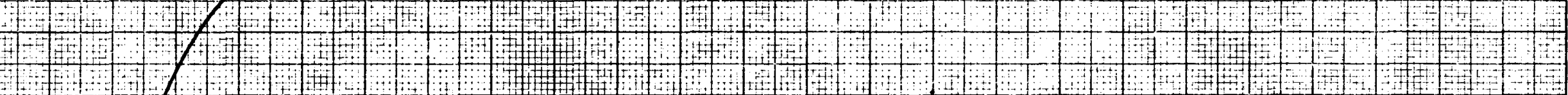

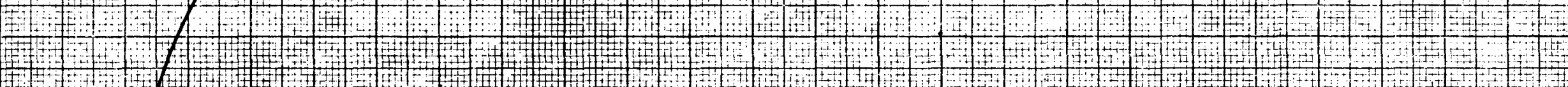
(1) 5 (1)

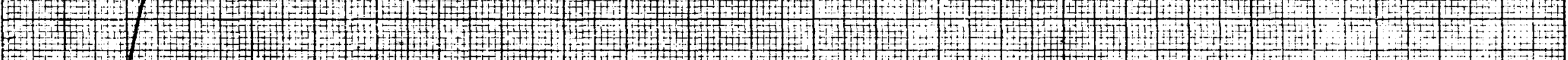

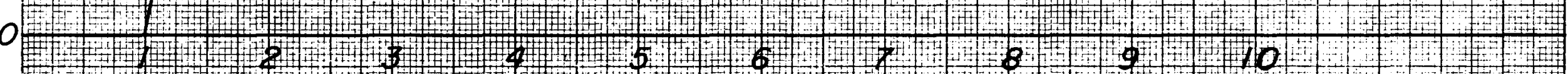

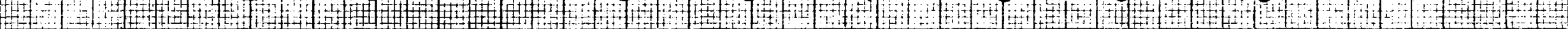

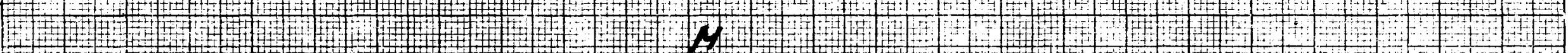
4 Hבt H

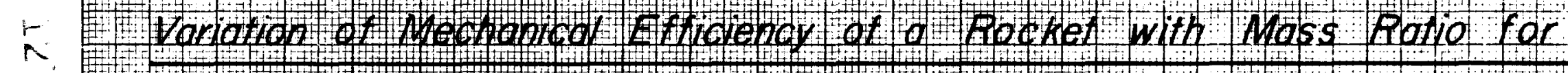

$\beth$

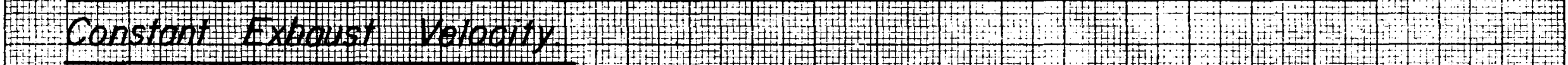
3

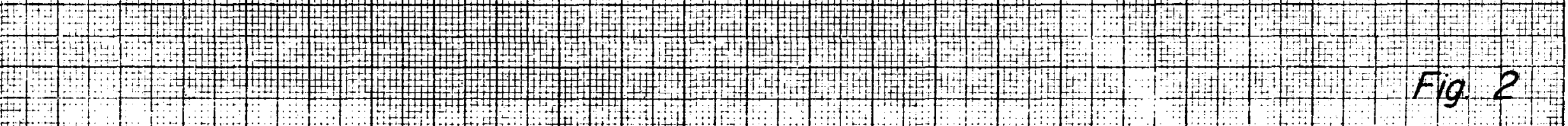




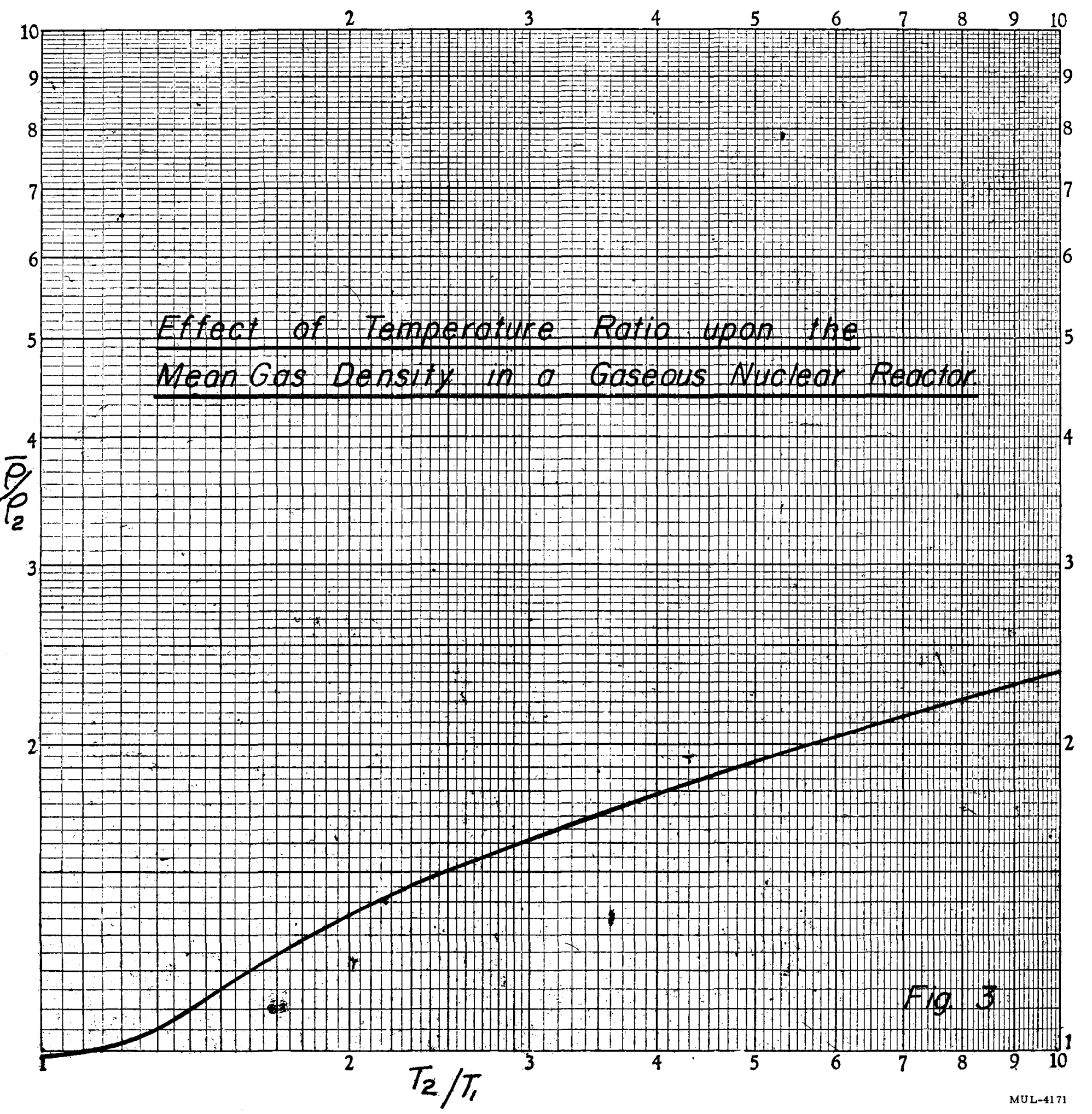




\section{0}

\section{0}

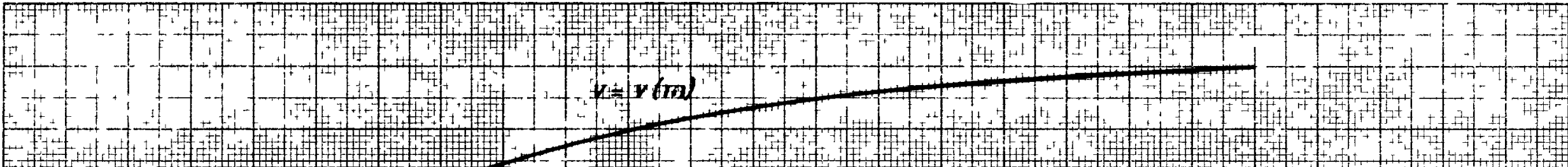

.5 $+4+4$

?,

\section{0}

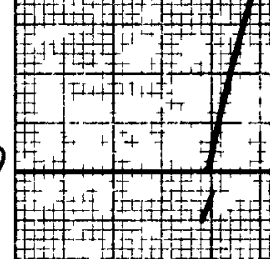

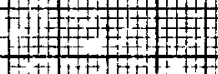

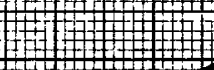

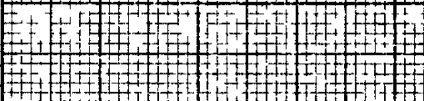

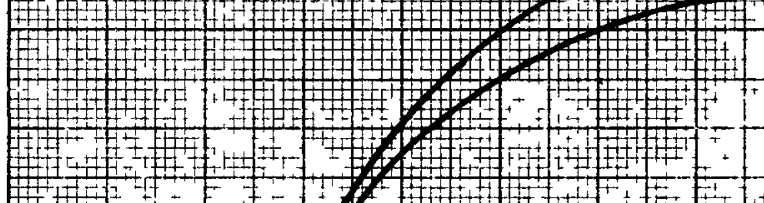

I1

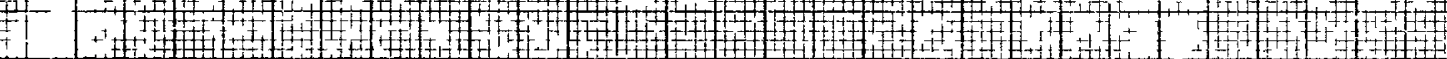

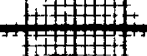
$4+4 \cos$

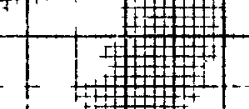
T.

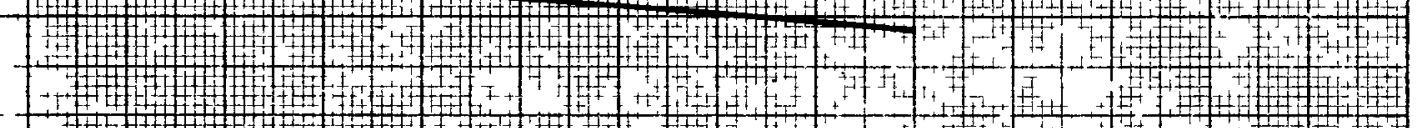
(4) 1

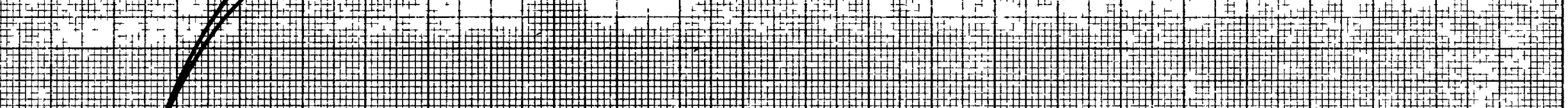
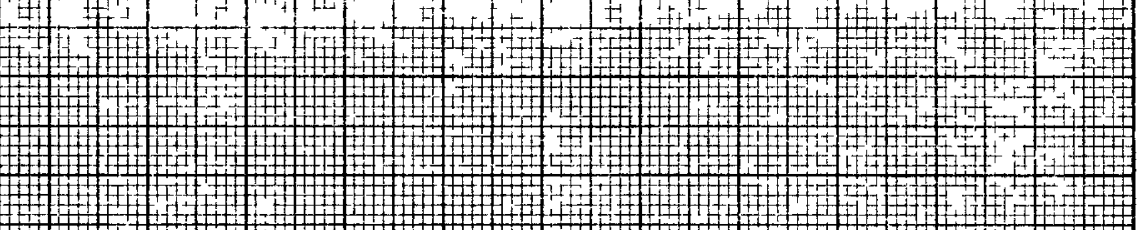

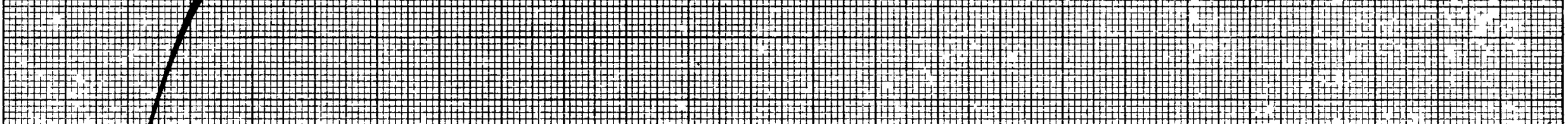
(I)

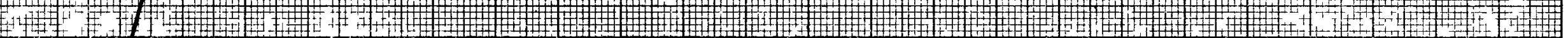

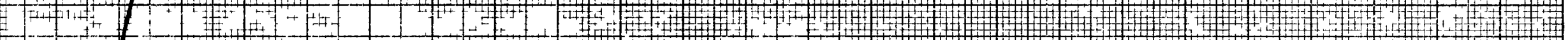

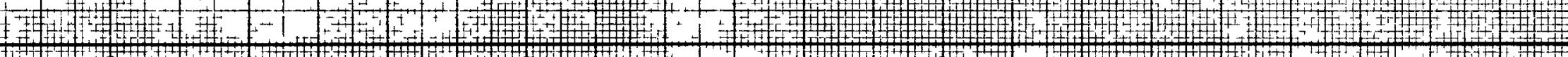
$(3+1+16$

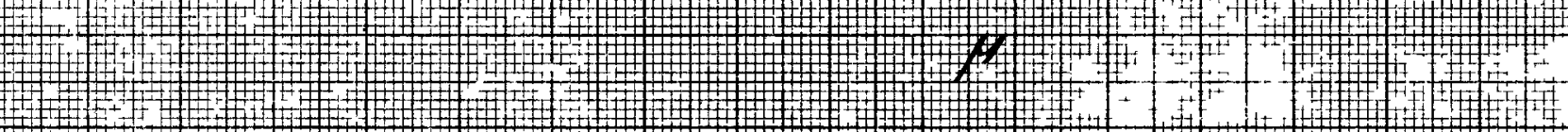
(2)

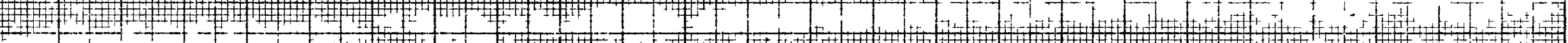

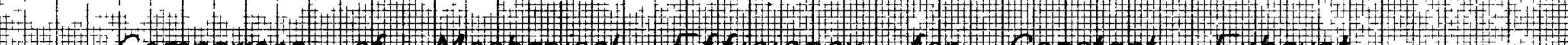

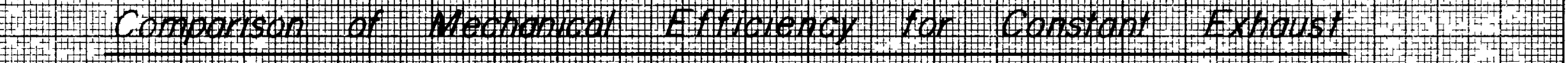

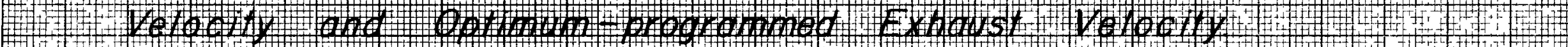

(2) WI 


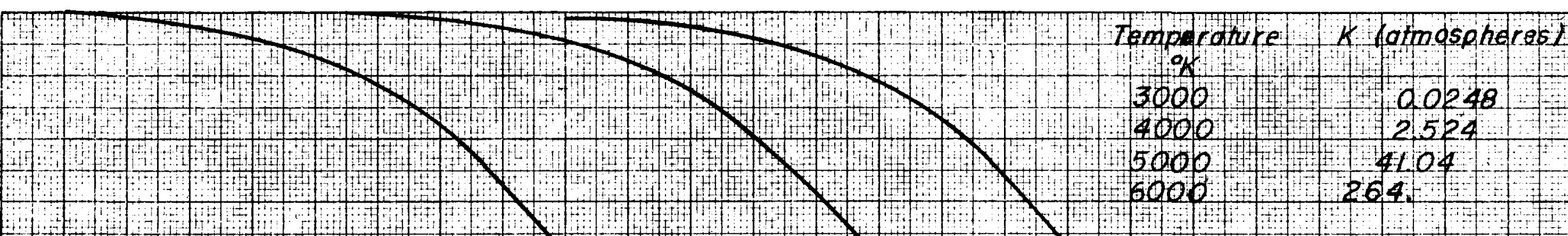

$+$
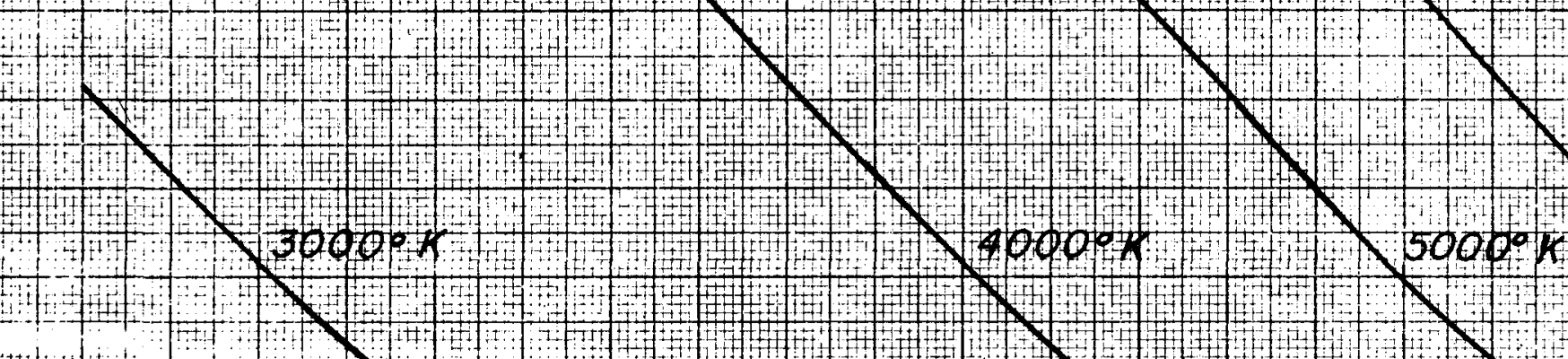


\section{0}

180

160

(1)

(1) (1) W W

140

$h$

120

$\mathrm{rcal}$

100

80

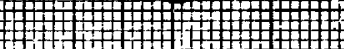

W

60

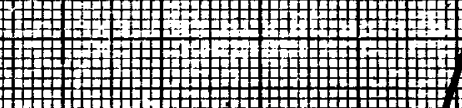

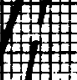

(2) (2)

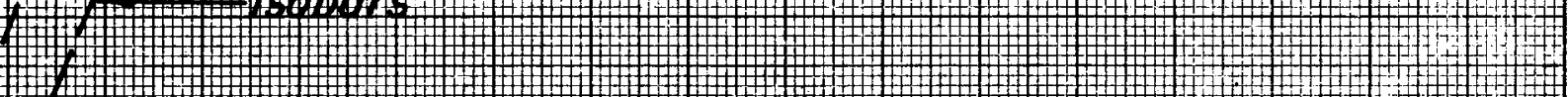
$(\ldots$ 


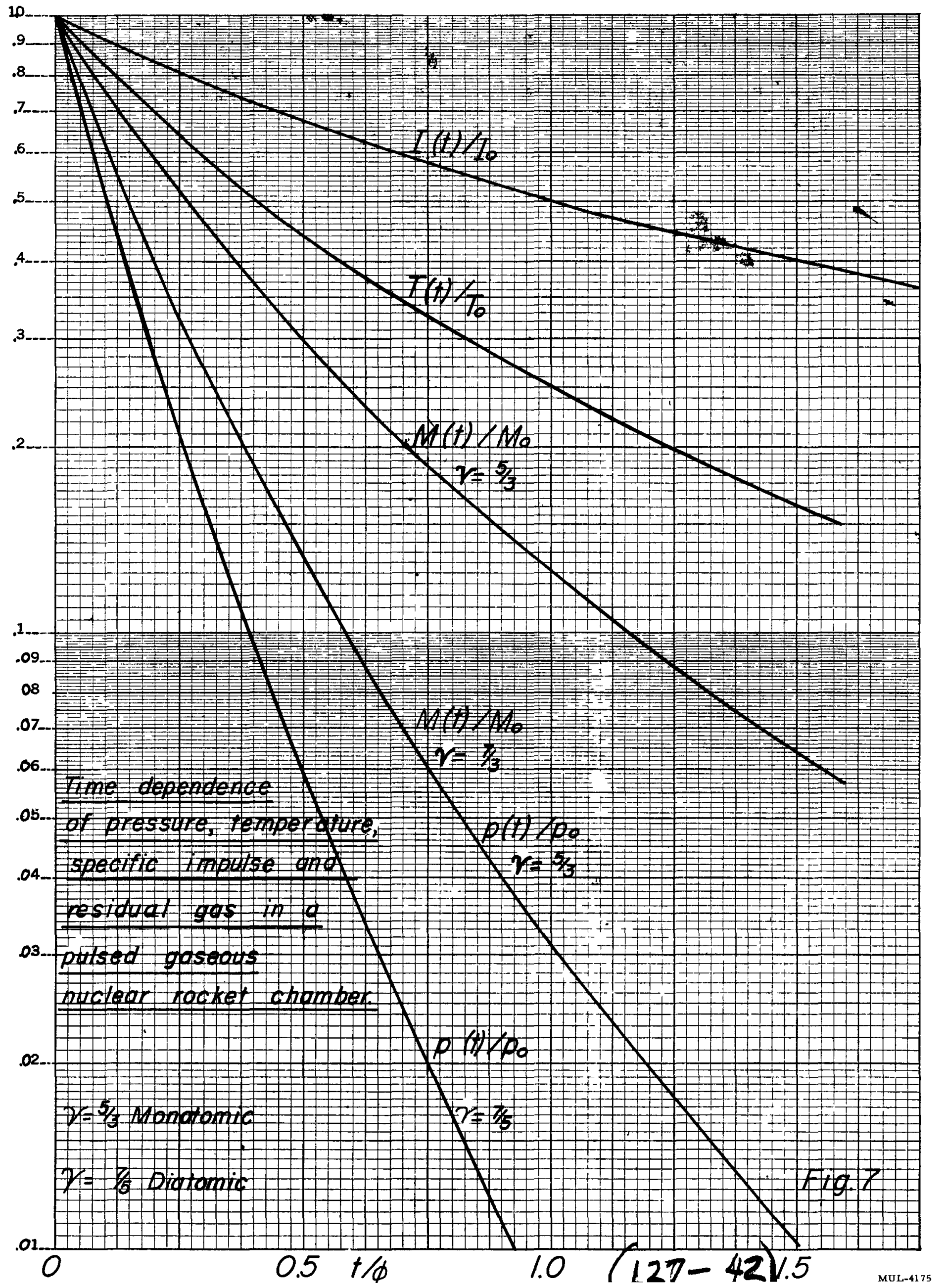

